\title{
Mitophagy-dependent macrophage reprogramming protects against kidney fibrosis
}

Divya Bhatia, ${ }^{1}$ Kuei-Pin Chung, ${ }^{2,3}$ Kiichi Nakahira, ${ }^{2}$ Edwin Patino, ${ }^{1}$ Michelle C. Rice, ${ }^{1}$ Lisa K. Torres, ${ }^{2}$ Thangamani Muthukumar, ${ }^{1,4}$ Augustine M.K. Choi, ${ }^{2,4}$ Oleh M. Akchurin, ${ }^{4,5}$ and Mary E. Choi ${ }^{1,4}$

'Division of Nephrology and Hypertension and 'Division of Pulmonary and Critical Care Medicine, Joan and Sanford I. Weill Department of Medicine, Weill Cornell Medicine, New York, New York, USA. ${ }^{3}$ National Taiwan University Hospital, Taipei, Taiwan. ${ }^{4}$ NewYork-Presbyterian Hospital, New York, New York, USA. ${ }^{5}$ Division of Pediatric Nephrology, Department of Pediatrics, Weill Cornell Medicine, New York, New York, USA.

Mitophagy, by maintaining mitochondrial quality control, plays a key role in maintaining kidney function and is impaired in pathologic states. Macrophages are well known for their pathogenic role in kidney fibrosis. Here, we report that PINK1/Parkin-mediated mitophagy in macrophages is compromised in experimental and human kidney fibrosis. We demonstrate downregulation of mitophagy regulators mitofusin-2 (MFN2) and Parkin downstream of PINK1 in kidney fibrosis. Loss of either Pink1 or Prkn promoted renal extracellular matrix accumulation and frequency of profibrotic/M2 macrophages. Pink1-/- or Prkn ${ }^{-/-}$BM-derived macrophages (BMDMs) showed enhanced expression of rictor. Mitochondria from TCF- $\beta 1$-treated Pink1 ${ }^{-1-}$ BMDMs exhibited increased superoxide levels, along with reduced respiration and ATP production. In addition, mitophagy in macrophages involves PINI1-mediated phosphorylation of downstream MFN2, MFN2-facilitated recruitment of Parkin to damaged mitochondria, and macrophage-specific deletion of $M f n 2$ aggravates kidney fibrosis. Moreover, mitophagy regulators were downregulated in human CKD kidney and TGF- $\beta 1$-treated human renal macrophages, whereas Mdivi1 treatment suppressed mitophagy mediators and promoted fibrotic response. Taken together, our study is the first to our knowledge to demonstrate that macrophage mitophagy plays a protective role against kidney fibrosis via regulating the PINK1/MFN2/Parkin-mediated pathway.

Conflict of interest: The spouse of MEC is a cofounder and shareholder and serves on the Scientific Advisory Board of Proterris Inc. AMKC is a cofounder and stockholder and serves on the Scientific Advisory Board for Proterris, which develops therapeutic uses for carbon monoxide. AMKC also has a use patent on (no. 7,678,390) carbon monoxide.

Copyright: () 2019, American Society for Clinical Investigation.

Submitted: August 19, 2019 Accepted: October 16, 2019 Published: December 5, 2019 .

Reference information: JCI Insight. 2019;4(23):e132826. https://doi.org/10.1172/jci. insight.132826.

\section{Introduction}

Macrophages play an integral role in inflammation and subsequent progression of kidney fibrosis, a common manifestation of chronic kidney disease (CKD) (1). Macrophage depletion in experimental models of kidney fibrosis such as unilateral ureteral obstruction (UUO) results in dramatically reduced interstitial fibrosis (2). Following kidney injury, tubular cell-derived chemokines, including C-C motif chemokine ligand 2 (CCL2), promote the infiltration of $\mathrm{C}-\mathrm{C}$ chemokine receptor type 2 (CCR2) expressing $\mathrm{Ly}_{6 \mathrm{C}} \mathrm{hi}$ /proinflammatory monocytes that express markers of $\mathrm{M} 1 /$ classically activated macrophages into the kidney $(3,4)$. The inflammatory renal microenvironment favors the switch-over of Ly6Chi/ proinflammatory or M1 population to Ly6 $\mathrm{C}^{\mathrm{lo}}$ /antiinflammatory phenotype, which exhibits a profibrotic transcriptional profile and may contribute to M2 macrophages $(5,6)$.

Persistent kidney injury and inflammation result in the failure of tissue repair, mitochondrial dysfunction, and tubular apoptosis $(1,6,7)$. Chronic unresolved inflammation also promotes switchover of proinflammatory macrophages into antiinflammatory or M2 phenotype (7), which results in increased TGF- $\beta 1$ production (8). TGF- $\beta 1$, a pluripotent multifunctional and a key profibrogenic cytokine, favors the transdifferentiation of M2 macrophages into myofibroblasts $(7,8)$. Monocyte/ macrophage-specific deletion of TGF- $\beta$ receptor II has been shown to significantly reduce the infiltration of macrophages and tubulointerstitial fibrosis (9). However, macrophage-specific TGF- $\beta 1$ deletion failed to prevent kidney fibrosis $(10,11)$. Despite the increasing attention on the role of macrophages in kidney fibrosis, the mechanism of macrophage-induced extracellular matrix (ECM) remodeling is poorly understood. 
Mitochondrial dysfunction has emerged as a critical contributor to various diseases, including metabolic, cardiovascular, neurodegenerative, and neuromuscular diseases (12). The kidney has the highest number of mitochondria (13), second to the heart, to fulfill its energy demands for efficient electrolyte reabsorption, active transport, and removal of metabolic wastes (14). Therefore, the mitochondrial quality control through the removal of dysfunctional mitochondria is essential for cellular homeostasis and the maintenance of kidney function (13). The failure of elimination of damaged or superfluous mitochondria contributes to increased oxidative stress $(12,13)$. Mitophagy is the selective mitochondrial autophagy, which is triggered by excessive oxidative stress due to increased mitochondrial-derived ROS (mROS) levels and plays a critical role in the recycling of damaged mitochondria (15).

The initiation of mitophagy involves tagging and fragmentation of dysfunctional mitochondria for sequestration in the mitophagosome that is chiefly controlled by 2 regulatory proteins: phosphatase and tensin homolog-induced kinase1 (PINK1; a serine/threonine kinase) and Parkin (E3 ubiquitin ligase, encoded by Prkn). Mitochondrial damage favors the accumulation of PINK1 on depolarized mitochondria (16). PINK1 promotes the recruitment of the cytosolic Parkin to the affected mitochondria $(17,18)$. Subsequent ubiquitination of outer mitochondrial membrane (OMM) proteins by E3 ligase activity of Parkin tags damaged mitochondria for degradation via recruitment of the autophagy adaptor p62/sequestosome 1 (p62/ SQSTM1) and microtubule-associated protein light chain 3 (LC3), which results in an eventual turnover of dysfunctional mitochondria (17-19). In the case of cardiomyocytes, mitophagy involves PINK1-mediated phosphorylation of mitofusin-2 (MFN2), an OMM protein that functions as a mitochondrial receptor for Parkin and promotes mitophagy by facilitating the recruitment of Parkin to the impaired mitochondria (20). Deletion of MFN1 and MFN2 suppressed mitophagy in mouse embryonic fibroblasts and cardiomyocytes $(20,21)$. Parkin, by ubiquitination of several OMM proteins including MFN1 and MFN2 (18, 19), plays a critical role in the regulation of mitophagy (15-17).

The underlying molecular mechanism of mitophagy in macrophages and its role during progressive kidney diseases remain poorly understood. Tang et al. recently reported findings of increased renal tubular damage in single and double Pink1- and Prkn-KO mice in a model of ischemic acute kidney injury (22). However, the functional role of macrophage mitophagy in the pathogenesis of kidney fibrosis, the common pathway in CKD, has not been previously investigated. Using 2 independent experimental mouse models of kidney fibrosis, and primary mouse and human renal macrophages, we uncovered a cytoprotective role of PINK1/MFN2/Parkin-dependent mitophagy against macrophage-induced fibrotic response during the progression of kidney fibrosis. Moreover, mitophagy regulators were downregulated in the human CKD kidney. We further demonstrate that the deficiency of mitophagy regulators results in macrophage reprogramming toward a profibrotic/M2 phenotype and promotes kidney fibrosis.

\section{Results}

Mitophagy regulators Parkin and MFN2, downstream of PINK1, are downregulated in kidney fibrosis. To investigate the role of PINK1/Parkin-mediated mitophagy during kidney fibrosis, we utilized a well-established murine model of kidney fibrosis induced by UUO. The expression of MFN2 (84 kDa) and Parkin (52 kDa), which are downstream regulators of PINK1-mediated mitophagy (15), was decreased in the kidneys of WT mice 7 days after UUO compared with sham surgery (Figure 1A). The expression of LC3-II, a lipidated form of LC3 that wraps around the mitochondria and promotes mitophagosome formation (15-17), was also decreased after UUO (Figure 1A).

We employed an alternative model of kidney fibrosis induced by adenine diet (AD) and confirmed similarly reduced expression of MFN2, Parkin, and LC3-II in the kidneys from mice fed with AD compared with control diet for 14 days (Supplemental Figure 1A; supplemental material available online with this article; https://doi.org/10.1172/jci.insight.132826DS1). These findings in both experimental models indicate that the expression of downstream regulators of PINK1-mediated mitophagy decreased during kidney fibrosis.

Loss of PINK1 or Parkin aggravates macrophage-derived fibrotic response and kidney fibrosis. To understand the functional role of PINK1/Parkin-mediated mitophagy in kidney fibrosis, we compared the fibrotic response in the kidneys between PINK1-deficient (Pink1 $1^{-/-}$) and Parkin-deficient $\left(\right.$Prkn $\left.^{-/-}\right)$mice and their corresponding littermate WT $\left(\mathrm{Pink1}^{+/+}\right.$and $\left.\mathrm{Prkn}^{+/+}\right)$mice 7 days after UUO or sham surgery. We found that the Pink1 ${ }^{-1-}$ and $\mathrm{Prkn}^{-/-}$mice both displayed higher collagen deposition in the obstructed kidneys compared with WT mice, as assessed by Masson's trichrome staining (Figure 1B). Moreover, kidneys from Pink1 ${ }^{-/-}$and $\mathrm{Prkn}^{-/-}$mice showed higher protein expression of profibrotic markers, 

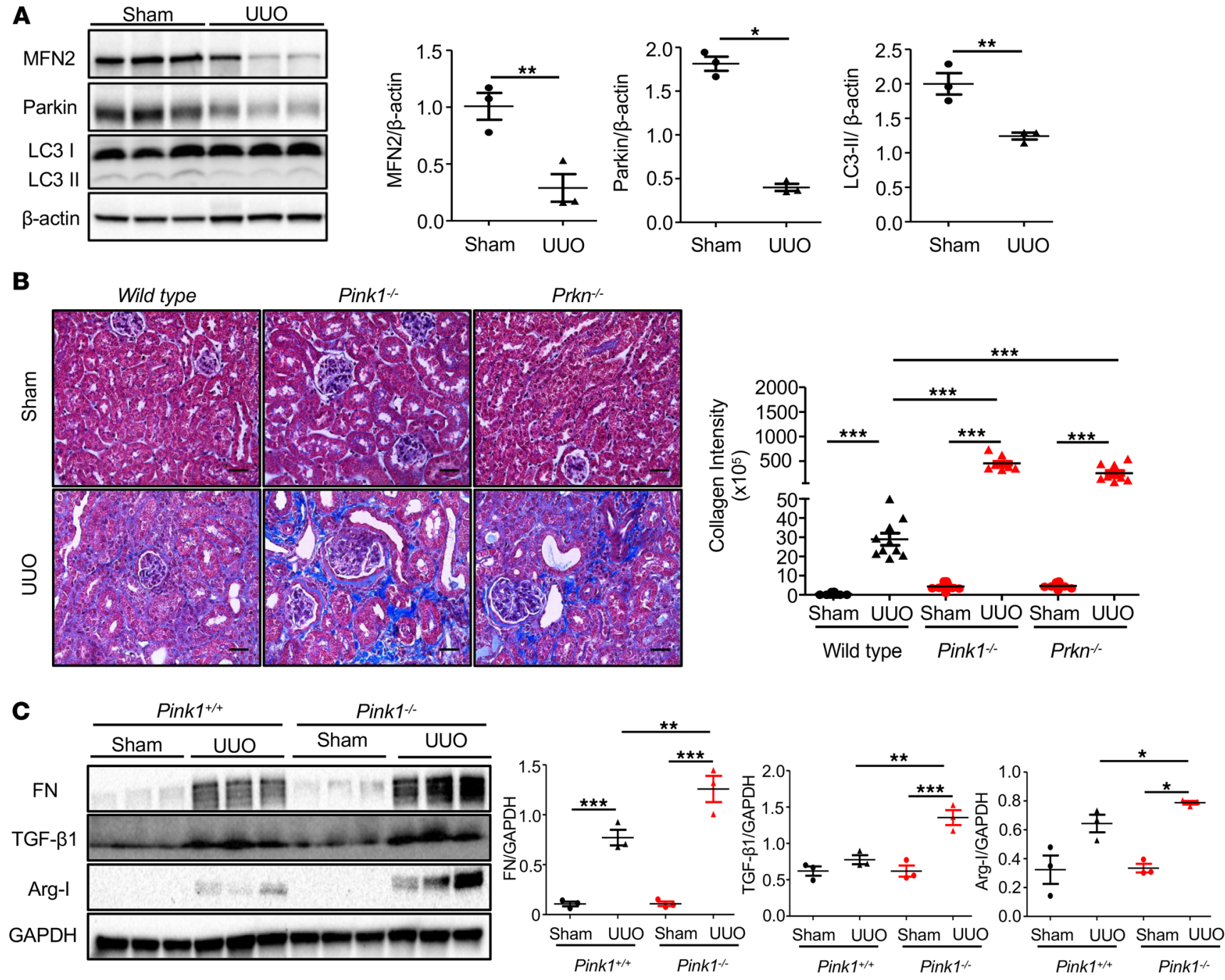

D
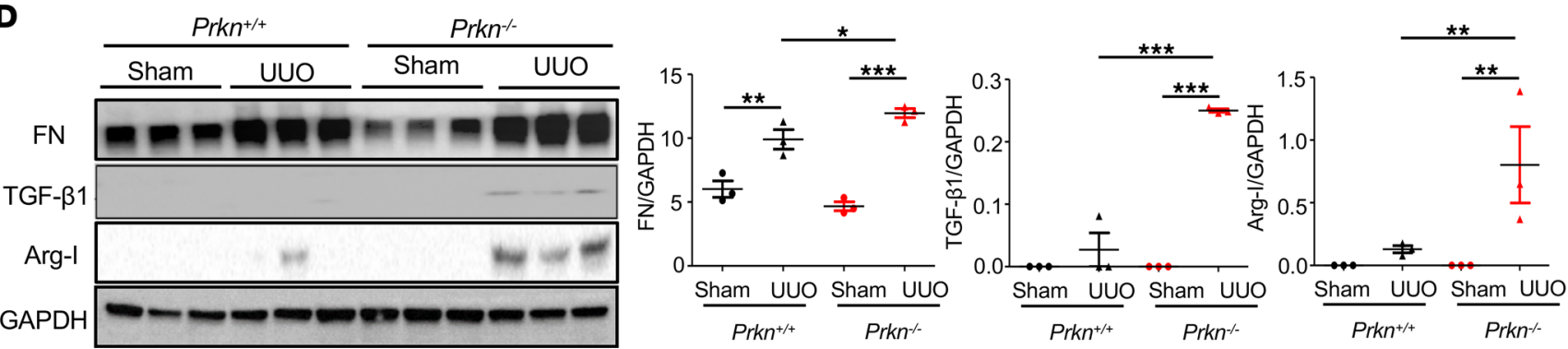

Figure 1. Mitophagy-related proteins are downregulated in experimental kidney fibrosis, and loss of PINK1 or Parkin promotes kidney fibrosis. (A) Western blot and densitometry analysis for expression of Mitofusin 2 (MFN2), Parkin, and microtubule-associated protein light chain 3 (LC3) normalized to $\beta$-actin in kidney tissue lysates from WT mice 7 days after sham or UUO surgery. Data are mean \pm SEM representative of 3 independent experiments ( $n=3$ per group) and analyzed by Student's unpaired 2-tailed $t$ test. (B) Representative Masson's trichrome-stained kidney tissue sections ( $\times 40$ magnification) from WT, Pink $1^{1-}$, and $P r k n^{-1-}$ mice 7 days after sham ( $n=3$ per group) or UUO ( $n=5$ per group) surgery. Ten areas from random fields per experimental group were analyzed and quantitated using Image). Data are mean \pm SEM, compared using 1-way ANOVA. Scale bars: $200 \mu \mathrm{m}$. (C and D) Western blot and densitometry analysis for the expression of fibronectin (FN), TCF- $\beta 1$, and arginase I (Arg-I) normalized to GAPDH in kidney tissue lysates from Pink ${ }^{1^{+/}}$and Pink $1^{-/-}$(C), as well as $\mathrm{Prkn}^{+/+}$and $\mathrm{Prkn}^{-/-}$(D), mice 7 days after sham or UUO surgery. Data are mean \pm SEM representative of 3 independent experiments ( $n=3$ per group) and analyzed by 1 -way ANOVA. ${ }^{*} P<0.05,{ }^{*} P<0.01,{ }^{* *} P<0.001$. 
fibronectin (FN, $220 \mathrm{kDa}$ ), TGF- $\beta 1$ (cleaved, $12.5 \mathrm{kDa}$ ), and M2/profibrotic macrophage marker arginase I (Arg-I, $35 \mathrm{kDa}$ ) after UUO than Pink1 ${ }^{+/+}$(Figure 1C) and $\mathrm{Prkn}^{+/+}$(Figure 1D) mice.

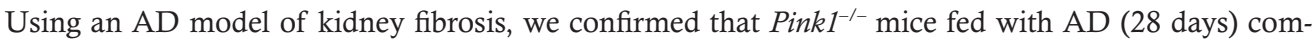
pared with Pink1 $^{+/+}$AD-fed mice displayed higher kidney collagen content, as confirmed by quantitation of hydroxyproline content (Supplemental Figure 1B), and lower kidney weight (Supplemental Figure 1C), suggesting higher renal damage since the kidney weight negatively correlates with the degree of kidney damage (23). Circulating levels of CCL2 (also known as monocyte chemoattractant protein-1; MCP-1) are directly related to the monocyte infiltration, inflammation, and tubulointerstitial fibrosis (24), and AD-fed Pink1 $1^{-/}$mice displayed higher plasma levels of CCL2 than AD-fed Pink1 ${ }^{+/+}$mice (Supplemental Figure 1D), suggesting higher monocyte recruitment into the damaged kidney. Further, we observed that the kidneys from AD-fed $\mathrm{Prkn}^{-/}$mice also displayed higher expression of FN, collagen-I (Col-I, $115 \mathrm{kDa}$ ), and profibrotic macrophage markers CD206 (190 kDa) and galectin-3 (Gal-3) compared with AD-fed Prkn ${ }^{+/+}$ mice (Supplemental Figure 1E). In addition, we demonstrated that the counts of $\mathrm{Gal}-3^{+} \mathrm{F} 4 / 80^{+}$renal macrophages and TGF- $\beta 1^{+} \mathrm{F} 4 / 80^{+}$renal macrophages were increased in the kidneys of mice fed with $\mathrm{AD}(28$ days) compared with control diet, as assessed by flow cytometry analysis of renal single-cell suspensions and gated on $\mathrm{CD}^{+} 5^{+}$side scatter low $\left(\mathrm{SSC}^{\mathrm{lo}}\right.$ ) mononuclear cells (Supplemental Figure 1, F and G). We further confirmed that the Gal- $3^{+} \mathrm{F} 4 / 80^{+}$renal macrophages were higher in the AD-fed Pink1 ${ }^{-1-}$ and Prkn ${ }^{-1-}$ mice compared with the $\mathrm{AD}$-fed $\mathrm{Pink1}^{+/+}$and $\mathrm{Prkn}^{+/+}$mice, respectively.

Taken together, the above findings from 2 independent experimental models of CKD suggest that PINK1 and Parkin exert protective functions against kidney fibrosis.

PINK1 or Parkin deficiency amplifies frequency of renal profibrotic macrophages. To further investigate the involvement of PINK1 in modulating macrophage-derived fibrotic response during kidney fibrosis, we first determined the frequency of circulating and renal monocyte/macrophage populations after UUO. We compared the numbers of total mononuclear $\left(\mathrm{CD} 45^{+} S S C^{10}\right)$ and phagocytic $\left(\mathrm{F} 4 / 80^{+} \mathrm{CD} 45^{+}\right)$populations and found similar increases in the absolute counts of both total mononuclear (Supplemental Figure 2A) and mononuclear phagocytic (Supplemental Figure 2B) cells in the obstructed kidneys from Pink $1^{+/+}$ and Pink1 $1^{-/}$mice after UUO. The absolute numbers of profibrotic/M2 macrophages were determined by gating on $\mathrm{CD} 206^{+} \mathrm{F} 4 / 80^{+}$cells. The profibrotic/M2 macrophages in the kidneys also increased after UUO; however, the obstructed kidneys from $P$ ink $1^{-1-}$ mice had significantly higher numbers of profibrotic/M2 macrophages than Pink1 ${ }^{+/+}$mice (Figure 2A).

The differential expression of Ly6C is related to specific monocyte phenotype $(5,25-27)$. Based on its differential expression, we gated 3 populations of CD11b expressing Ly6C hi, Ly6 $C^{\text {int }}$, and Ly6C ${ }^{\text {lo }}$ monocytes. The counts of proinflammatory $\left(\mathrm{Ly} 6 \mathrm{C}^{\mathrm{hi}} \mathrm{CD} 11 \mathrm{~b}^{+}\right)$monocytes in the kidneys after UUO increased in the Pink1 ${ }^{+/+}$mice (Figure 2B). However, compared with $\mathrm{Pink1}^{+/+}$mice, Pink $1^{-/-}$mice exhibited a lower frequency of the proinflammatory monocytes in the kidney. The frequency of $\mathrm{Ly} 6 \mathrm{C}^{\mathrm{int}} \mathrm{CD} 11 \mathrm{~b}^{+}$population, which is known to play reparative functions after kidney injury (25), was lower in the kidneys of Pink $1^{-1}$ than $P i n k 1^{+/+}$mice. Moreover, the obstructed kidneys from Pink1 $1^{-/-}$mice also had higher numbers of the Ly6 $\mathrm{C}^{\mathrm{lo}} \mathrm{CD} 11 \mathrm{~b}^{+}$monocytes compared with $\mathrm{Pink}^{1^{+/+}}$mice (Figure 2B). Lower frequency of Ly6 $\mathrm{Ch}^{\text {hi }}$ and high er numbers of Ly6 $\mathrm{C}^{\mathrm{lo}}$ phenotype in the obstructed kidneys from Pink1 $1^{-1-}$ mice suggest a potential switchover of Ly6C $\mathrm{C}^{\text {hi }}$ to $\mathrm{Ly} 6 \mathrm{C}^{\text {lo }}$ phenotype. There was no difference in the frequency of circulating proinflammatory $\left(\mathrm{Ly} 6 \mathrm{C}^{\text {hi }} \mathrm{CD} 11 \mathrm{~b}^{+}\right.$) monocytes between Pink1 ${ }^{+/+}$and Pink1 $1^{-/-}$mice after UUO (Supplemental Figure 2C). However, the numbers of circulating $\mathrm{Ly} 6 \mathrm{C}^{\mathrm{lo}} \mathrm{CD} 11 \mathrm{~b}^{+}$monocytes after UUO were higher in Pink1 ${ }^{\text {- }^{-}}$mice (Supplemental Figure 2D). The Ly6 $\mathrm{C}^{10} \mathrm{CD} 11 \mathrm{~b}^{+}$monocytes exhibit profibrotic transcriptional profile and favor the progression of kidney fibrosis through paracrine signaling (25-27), which could at least in part account for the higher numbers of the profibrotic/M2 macrophages in the fibrotic kidney of Pink $1^{-1-}$ mice.

We next sorted the renal macrophages after sham or UUO surgery. The Pink1 ${ }^{-1-}$ renal macrophages after UUO showed significantly higher expression of FN, indicating higher profibrotic macrophage expression in case of PINK1 deficiency (Figure 2C).

We further examined whether genetic deficiency of Pink1 could exaggerate TGF- $\beta 1$-induced fibrotic response by macrophages. In response to TGF- $\beta 1$ treatment, Pink1 $1^{-1-}$ BM-derived macrophages (BMDMs) displayed higher expression of profibrotic markers, including FN, Arg-I, and Gal-3 (26 kDa) (Figure 2D). These observations confirm that PINK1 suppresses the macrophage-induced fibrotic response.

We next investigated the effect of loss of Parkin on the expression of profibrotic/M2 macrophages and Ly6C monocyte populations after UUO. Consistent with the findings in Pink1 $1^{-1-}$ mice, the increase in the 
A
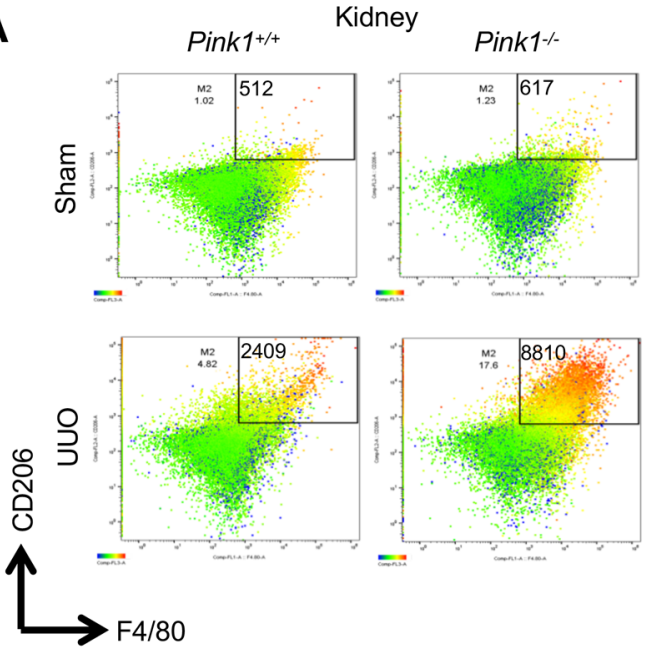

$F 4 / 80$

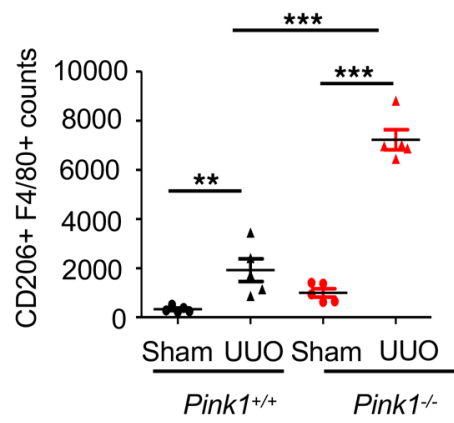

B
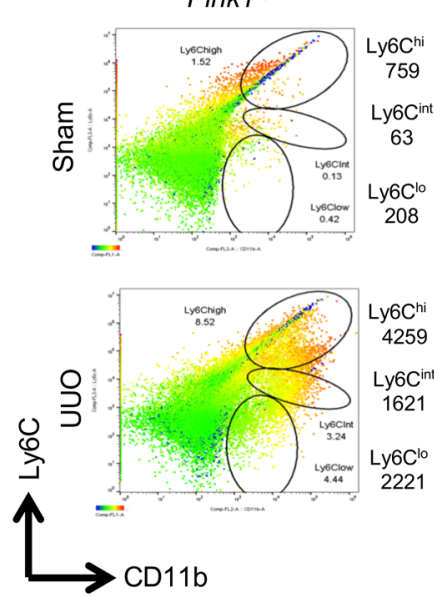

Kidney
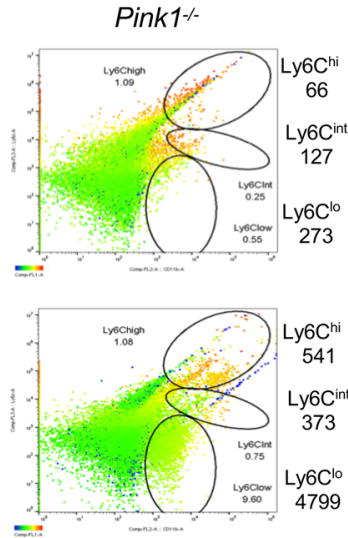
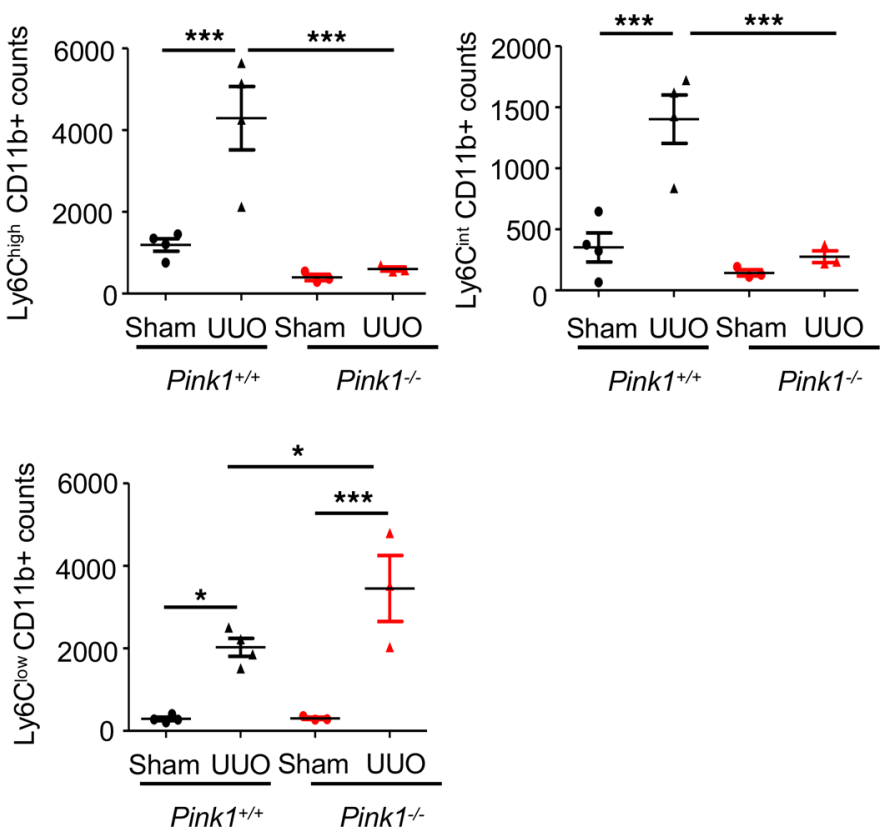

C Renal Macrophage

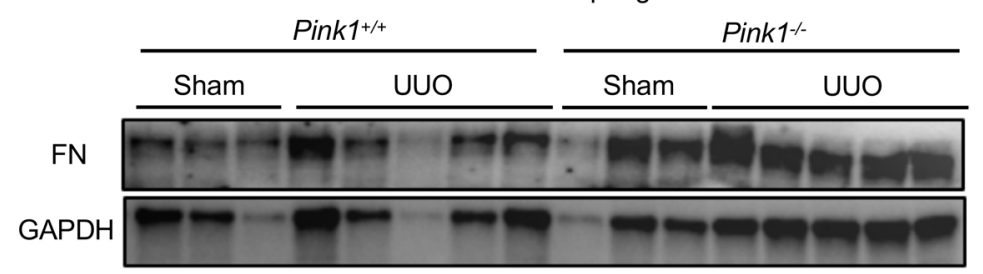

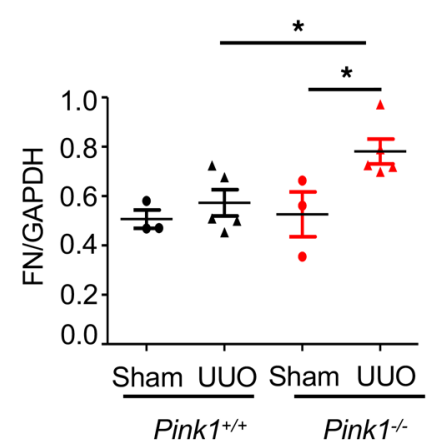

D

BMDMs

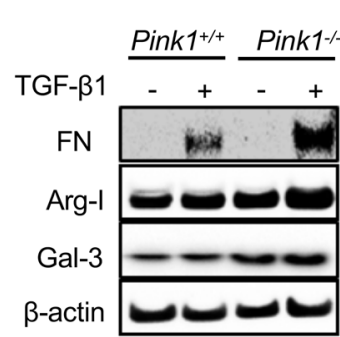

Figure 2. PINK1-deficient renal macrophages display higher profibrotic response. (A) Representative flow cytometric plots and analysis showing the numbers of CD206+F4/80+ cells in the kidney from Pink $1^{+/+}$and Pink $1^{-/-}$mice 7 days after sham or UUO surgery ( $n=5$ per group). (B) Representative flow cytometric data showing the counts of Ly6C $C^{\text {hi }}$, Ly $6 C^{\text {int }}$, and Ly6 $\mathrm{C}^{\circ 0} \mathrm{CD} 11 \mathrm{~b}^{+}$populations in the kidney from Pink ${ }^{+/+}(n=4$ per group) and Pink1 1-- ( $n=3$ per group) mice 7 days after sham or UUO surgery. (C) Densitometry analysis and Western blot for fibronectin (FN) normalized to GAPDH in Pink $1^{+/+}$and Pink $1^{-/-}$renal macrophages isolated 7 days after sham ( $n=3$ per group) or UUO ( $n=5$ per group) surgery. (D) Western blot for fibronectin (FN), arginase (Arg-I), galectin-3 (Gal-3), and $\beta$-actin in Pink1+/+ or Pink1 $1^{-/-}$BM-derived macrophages (BMDMs) cultured in the absence (-) or presence $(+)$ of TCF $-\beta 1(5 \mathrm{ng} / \mathrm{mL})$ for 24 hours. The data are representative of 3 independent experiments. Data are mean \pm SEM. ${ }^{*} P<0.05,{ }^{*} P<$ $0.01,{ }^{* *} P<0.001$ analyzed by 1 -way ANOVA. 

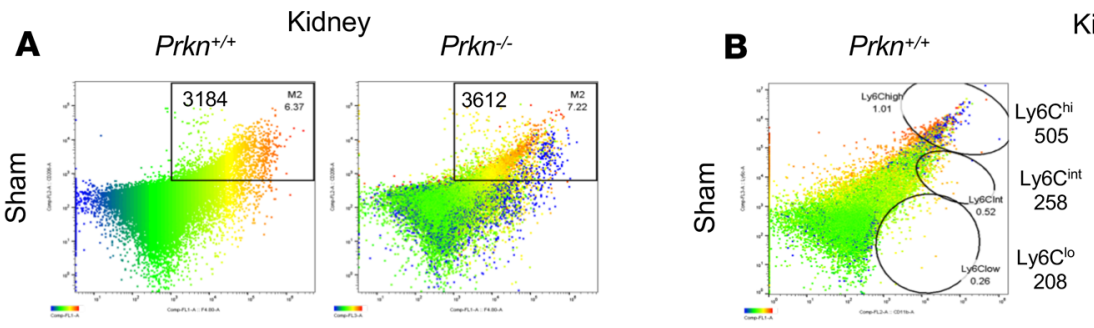

Kidney
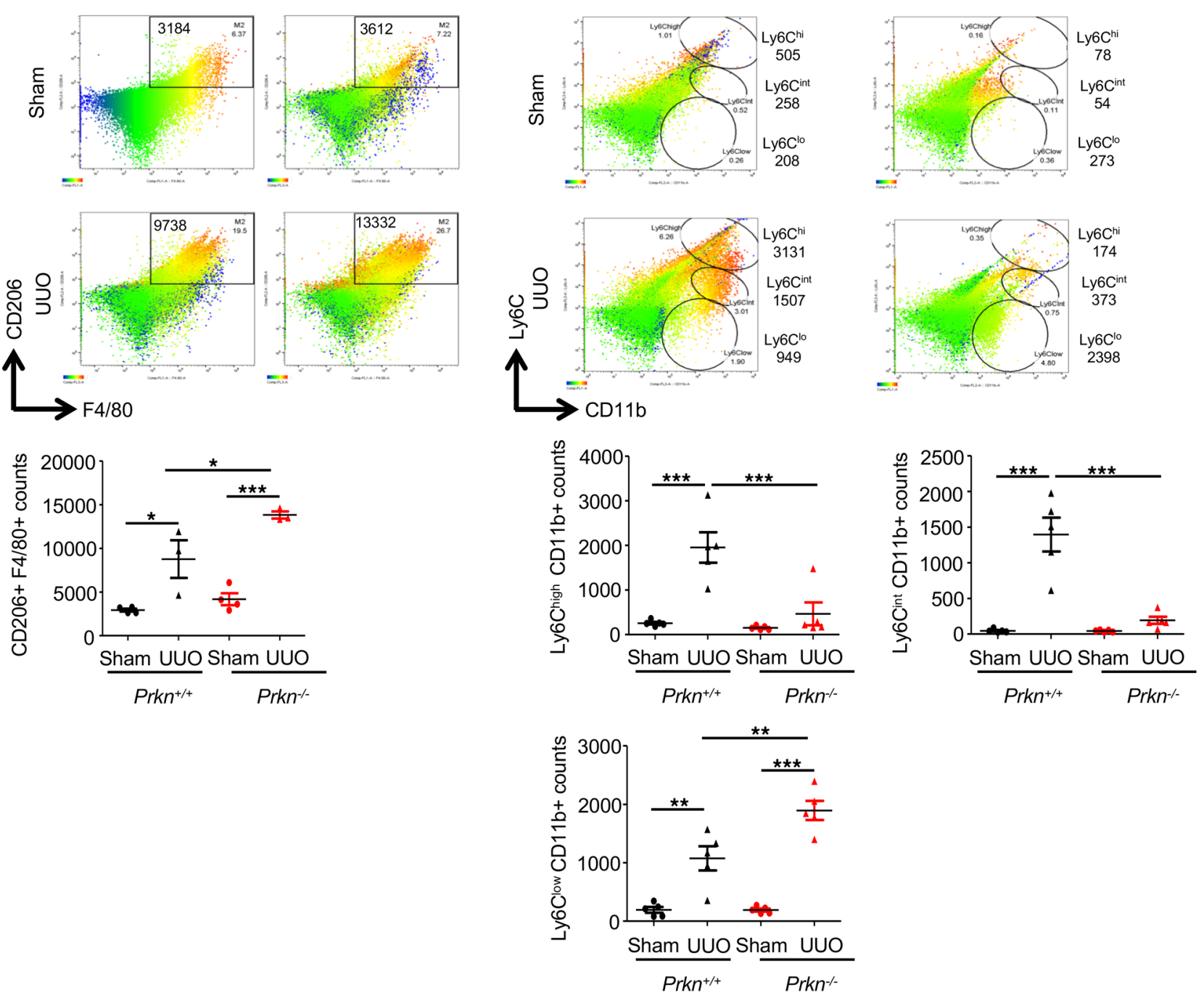

C

BMDMs

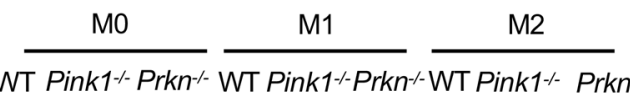

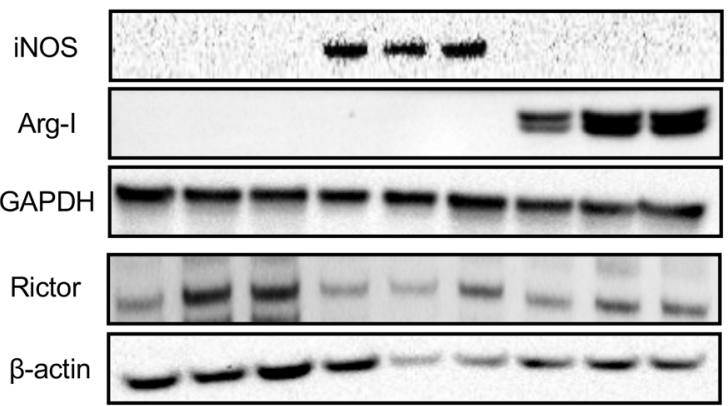

D

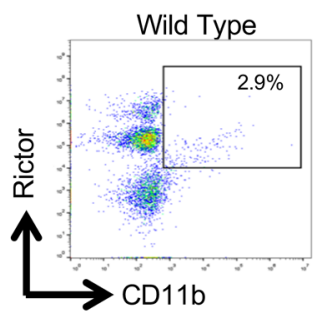

Wild Type

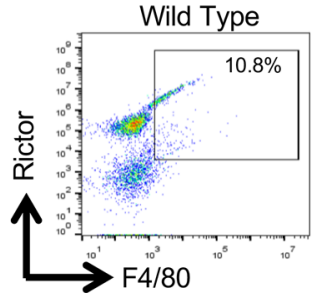

Peritoneal Cells

Pink1-1-

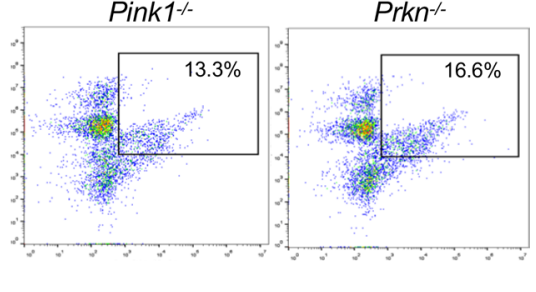

Pink1-

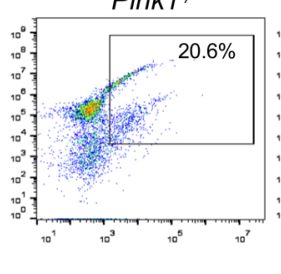

Prkn--

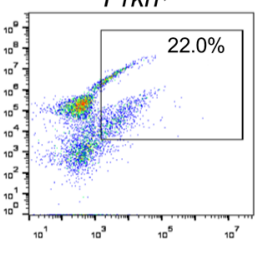

Figure 3. Loss of Parkin increases profibrotic macrophages during kidney fibrosis. (A) Flow cytometric data showing the numbers of $C D 206^{+} F 4 / 80^{+}$cells in the kidney from $\mathrm{Prkn}^{+/+}$and Prkn ${ }^{-/-}$mice 7 days after sham ( $n=4$ per group) or UUO surgery ( $n=3$ per group). (B) Frequencies of Ly6C $C^{\text {hi }}$, Ly6C $C^{\text {int }}$, and Ly6C ${ }^{\text {lo }}$ CD11b+ populations in the kidney from Prkn ${ }^{+/}$and Prkn ${ }^{-/-}$mice 7 days after sham ( $n=5$ per group) or UUO ( $n=5$ per group) surgery analyzed by flow cytometry. (C) Western blot for inducible nitric oxide synthase (iNOS), Arg-I, rictor, and GAPDH on MO, M1, or M2 WT, Pink1 $1^{-/}$, or Prkn ${ }^{-1-}$ BMDMs. The data are representative of 3 independent experiments. (D) Flow cytometry data showing the expression of rictor on $\mathrm{CD}_{11} \mathrm{~b}^{+}$(upper panels) or $\mathrm{F} 4 / 8 \mathrm{O}^{+}$(lower panels) populations in the cells isolated from peritoneal cavity. The data are representative of 3 independent experiments and are mean \pm SEM. ${ }^{*} P<0.05,{ }^{* *} P<0.01,{ }^{* *} P<0.001$ analyzed by 1 -way ANOVA 
absolute counts of profibrotic/M2 macrophages in the kidney 7 days after UUO was higher in $P_{r k n^{-/}}$mice than $\mathrm{Prkn}^{+/+}$mice (Figure 3A). The obstructed kidneys from $\mathrm{Prkn}^{-/-}$mice showed lower frequencies of both Ly6 $\mathrm{C}^{\text {hi }} \mathrm{CD} 11 \mathrm{~b}^{+}$and $\mathrm{Ly} 6 \mathrm{C}^{\text {int }} \mathrm{CD} 11 \mathrm{~b}^{+}$populations than $\mathrm{Prkn}^{+/+}$mice (Figure $3 \mathrm{~B}$ ). There were no differences in the UUO-induced increase in the numbers of total mononuclear (Supplemental Figure 3A) and mononuclear phagocytic (Supplemental Figure 3B) populations in the kidney and circulating Ly6Chi (Supplemental Figure 3C) monocytes between $\mathrm{Prkn}^{+/+}$and $\mathrm{Prkn}^{-/-}$mice. However, the frequencies of $\mathrm{Ly} 6 \mathrm{C}^{\mathrm{lo}} \mathrm{CD} 11 \mathrm{~b}^{+}$monocytes in the kidney (Figure 3B) and circulating Ly6 $6 \mathrm{C}^{\text {lo }}$ monocytes in the blood (Supplemental Figure 3D) after 7 days of UUO were higher in $\mathrm{Prkn}^{-/-}$mice compared with $\mathrm{Prkn}^{+/+}$mice. These results provide evidence that Parkin, downstream of PINK1, also prevents macrophage-derived fibrotic response in the kidney.

To understand the role of PINK1/Parkin-mediated mitophagy in macrophage polarization, we further induced the differentiation of BMDMs toward M0, M1, or M2 phenotypes in the presence of their respective inducers. Both Pink1 $1^{-/-}$and $\mathrm{Prkn}^{-/-}$BMDMs displayed greater ability to polarize toward the profibrotic/M2 phenotype (Figure 3C), as confirmed by higher expression of Arg-I and rictor (200 kDa). In addition, both Pink1 $^{-/-}$and $\mathrm{Prkn}^{-/-} \mathrm{CD} 1 \mathrm{~b}^{+}$monocytes and $\mathrm{F} 4 / 80^{+}$macrophages isolated from peritoneal cavity also showed higher expression of rictor (Figure 3D). The increase in the expression of rictor is known to play a critical role in the differentiation of M2 macrophages (28, 29). In contrast, loss of either PINK1 or Parkin did not affect the expression of inducible nitric oxide synthase-expressing (iNOS-expressing; $130 \mathrm{kDa}$ ) M1 macrophages. These findings indicate that the deficiency of either PINK1 or Parkin favors reprogramming of macrophages toward profibrotic/M2 phenotype.

Myeloid-specific deletion of Mfn2 exaggerates kidney fibrosis. To elucidate the macrophage-specific role of mitophagy in the progression of kidney fibrosis, we used $\mathrm{LysM}-\mathrm{Cr} \mathrm{e}^{+/-} \mathrm{Mfn} 2^{f / / f}$ mice, in which Cre recombinase activity was induced under myeloid-specific promoter lysozyme M (LysM). We observed that the kidneys from AD-fed $L y s M-C r e^{+/-} M f n 2^{f / f l}$ mice displayed higher expression of FN, CD206, TGF- $\beta 1$, and Gal-3 compared with their respective control ( $\left.L y s M-C r e^{-/-} M f n 2^{f / f f}\right)$ mice (Figure 4A). Even the basal level of $\mathrm{FN}$ expression in the kidney was higher in $L y s M-C r e^{+/-} M f n 2^{f l / f l}$ mice. AD-fed $L y s M-C r e^{+/-} M f n 2^{f l f l}$ mice also displayed higher collagen deposition and frequency of the $\mathrm{Ly} 6 \mathrm{C}^{\mathrm{lo}} \mathrm{CD} 11 \mathrm{~b}^{+}$monocytes in the kidneys compared with $L y s M-C r e^{-/-} M f n 2^{f l / f}$ mice, as assessed by Masson's trichrome staining (Figure 4B) and flow cytometry (Figure 4C), respectively. These findings indicate that macrophage-specific deletion of mitophagy regulator $M f n 2$ aggravates kidney fibrosis and suggest a critical role of macrophage mitophagy in protection against kidney fibrosis.

PINK1-mediated mitophagy is suppressed in renal macrophages after UUO and in TGF- $\beta 1$-treated macrophages. To investigate the role of the mitophagy in macrophages, we first determined the effect of TGF- $\beta 1$ treatment on PINK1 expression in THP-1-derived human macrophages. In our earlier studies, the detection of PINK1 expression in murine cells and tissues was limited due to lack of a reliable commercially available mouse-specific anti-PINK1 antibody. Using THP-1-derived human macrophages, we confirmed that PINK1 expression significantly decreased after TGF- $\beta 1$ treatment (Figure 5A). We next sorted the renal macrophages from WT mice after sham or UUO surgery (Supplemental Figure 4A) and determined that the expression of MFN2 and Parkin was significantly reduced in the macrophages isolated from the fibrotic kidney after UUO (Figure 5B).

We next determined the effect of TGF- $\beta 1$ on the recruitment of the mitophagy regulatory proteins to the mitochondria of macrophages. We isolated the mitochondrial fractions from the BMDMs cultured in the presence or absence of TGF- $\beta 1$ for 48 hours. TIM23 (a mitochondrial protein) and $\beta$-actin (a cytosolic marker) were used to confirm the purity of mitochondrial fractions. The expression of MFN2 and Parkin was significantly reduced in the mitochondrial lysates after TGF- $\beta 1$ treatment (Figure 5C). Furthermore, confocal microscopy revealed that the colocalization of MitoTracker red dye-stained mitochondria with autophagosome-specific marker LC3 was decreased in BMDMs after TGF- $\beta 1$ treatment (Figure 5, D and E). Pink $1^{-/-}$BMDMs displayed lower mitochondrial colocalization with LC 3 than Pink $1^{+/+}$BMDMs. We further performed the quantitative analysis of mitophagy via flow cytometry using renal single-cell suspensions isolated from kidneys of $P i n k 1^{+/+}$and $P i n k 1^{-/-}$mice fed with control or AD for 28 days. The mitophagy in macrophages was assessed by flow cytometry staining and by determining the median fluorescence intensity (MFI) of MitoTracker and LysoTracker dyes on F4/80+ cells. The mitophagy as

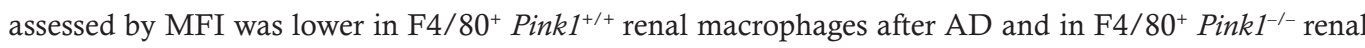
macrophages (Figure 5F). These data indicate that macrophage mitophagy is reduced in AD-induced kidney fibrosis and in Pink1-deficient renal macrophages. We observed similar results in Pink1 $1^{-/}$BMDMs. 

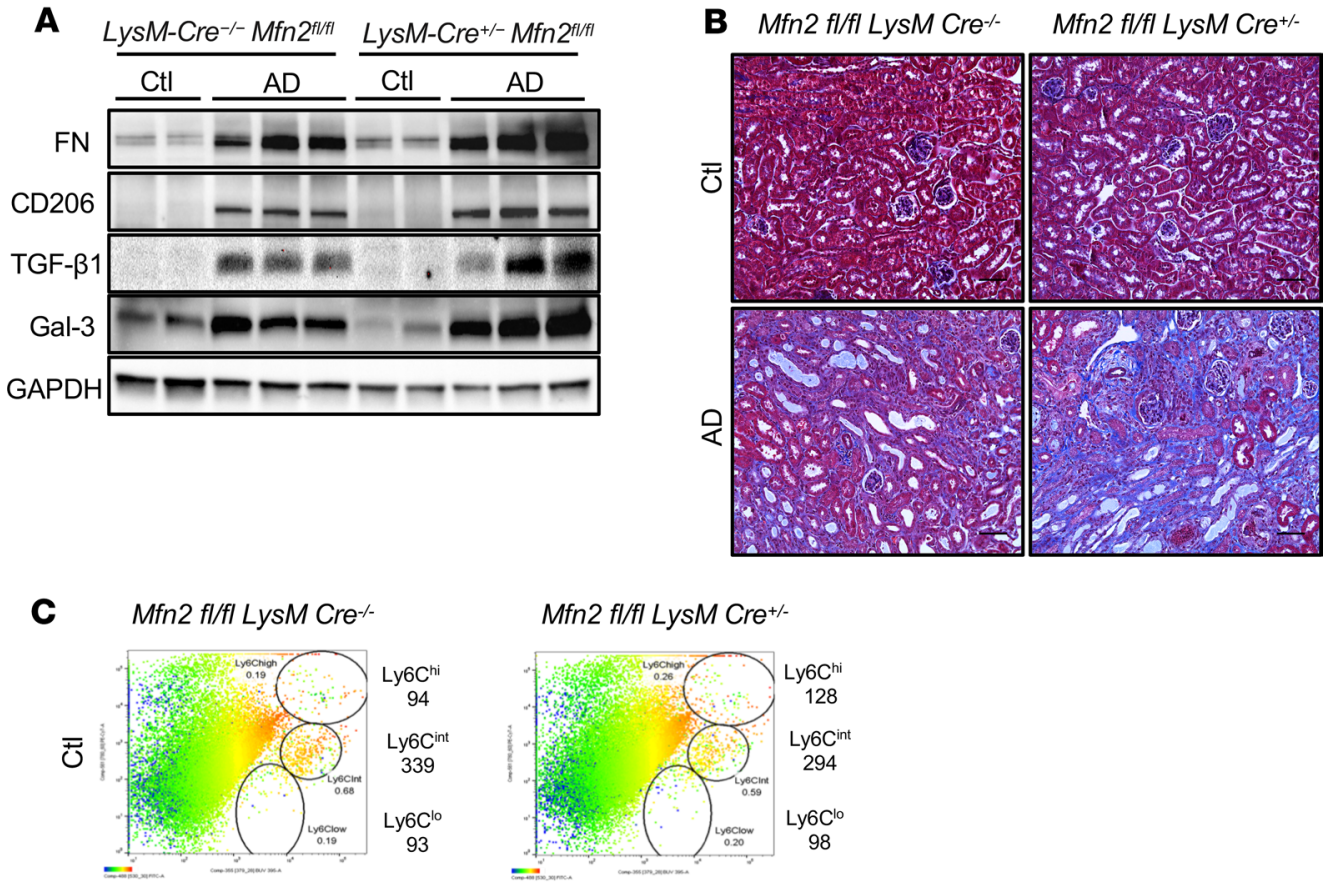

Mfn2 fl/fl LysM Cre Cr- $^{+/ 2}$
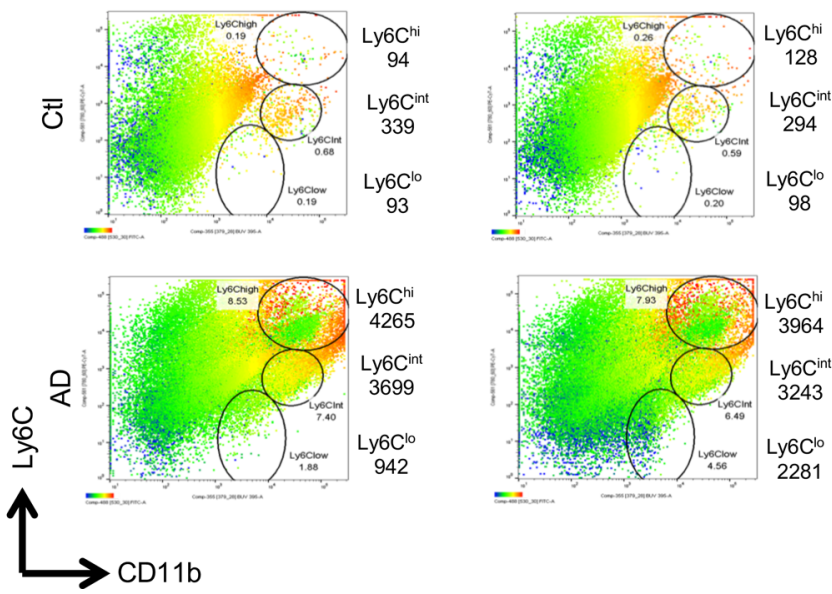

Figure 4. Myeloid-specific loss of MFN2 promotes kidney fibrosis. (A) Western blot for the expression of fibronectin

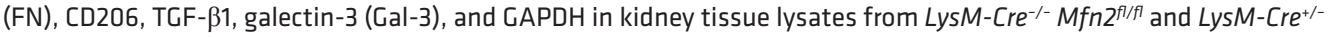
$M f n 2^{f / f l}$ mice fed with control (Ctl) or adenine (AD) diet for 28 days. (B) Representative Masson's trichrome-stained kidney tissue sections ( $\times 20$ magnification) from LysM-Cre ${ }^{-/-}$Mfn $2^{f / f / f}$ and $L y s M-C r e^{+/-} M f n 2^{f / f /}$ mice fed with Ctl or AD for 28 days. Scale bars: $200 \mu \mathrm{m}$. (C) Representative flow cytometric data showing the counts of Ly6Chi, Ly6Cint, and Ly6C ${ }^{10}$ $\mathrm{CD}_{11} \mathrm{~b}^{+}$populations in the kidney from $\mathrm{LysM}-\mathrm{Cre}^{-/-} \mathrm{Mfn} 2^{\mathrm{fl} / \mathrm{l}}$ and $\mathrm{LysM}-\mathrm{Cre}^{+/-} \mathrm{Mfn} 2^{\mathrm{fl} / \mathrm{l}}$ mice fed with $\mathrm{Ctl}$ or AD for 28 days. $n=2$ (CtI) or $n=3$ (AD) samples per group.

The mitophagy in BMDMs was detected by quantifying double-positive signals, confirming the fusion and colocalization of Mtphagy dye-stained mitochondria with Lyso dye-labeled lysosome. The mitophagy in BMDMs was reduced after TGF- $\beta 1$ treatment, and compared with Pink1 ${ }^{+/+}$BMDMs, Pink1 $1^{-/}$BMDMs displayed significantly reduced mitophagy (Figure 5, G and H) at the basal level. The above findings indicate that the mitophagy in macrophages is impaired under fibrotic conditions.

MFN2 is phosphorylated by PINK1 and promotes Parkin recruitment to mitochondria in macrophage mitophagy. PINK1 is a mitochondrial serine/threonine kinase capable of phosphorylating specific mitochondrial proteins, including MFN2 (20). We observed that knockdown of PINK1 expression in THP-1-derived human macrophages using PINK1-specific siRNA resulted in decreased expression of phosphorylated MFN2 at serine-442 (Ser-442, $86 \mathrm{kDa}$ ), relative to nontargeting control siRNA transfected macrophages (Figure 6A). TGF- $\beta 1$ treatment also reduced the expression of PINK1 (precursor or full length, $64 \mathrm{kDa}$ ) in THP-1derived human macrophages. These findings suggest that MFN2 is a downstream mitochondrial protein target substrate of PINK1 in macrophages.

We further observed that the $L y s M-C r e^{+/-} M f n 2^{f / f l}$ BMDMs from myeloid Mfn2-conditional KO mice displayed significantly lower mitophagy with or without TGF- $\beta 1$ treatment, compared with the corresponding control $L y s M-C r e^{-/-} M f n 2^{\ell / f} \mathrm{BMDMs}$ (Figure 6, B and C). These results indicate that MFN2 plays a critical functions in the regulation of mitophagy in macrophages. 
A
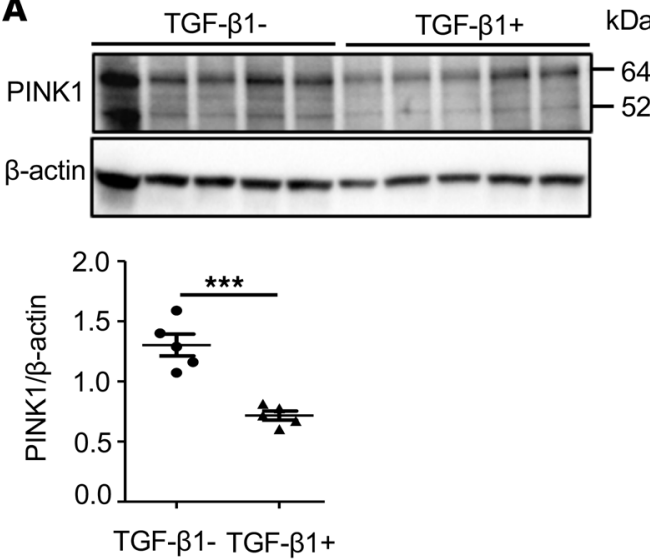

B
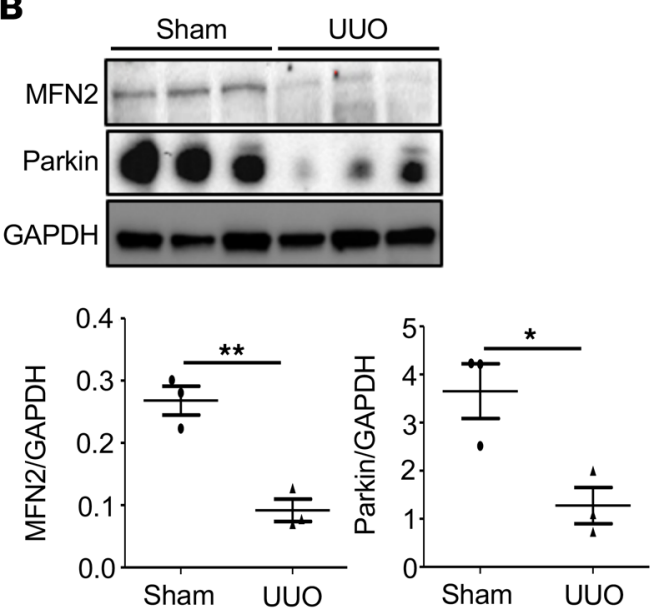

C
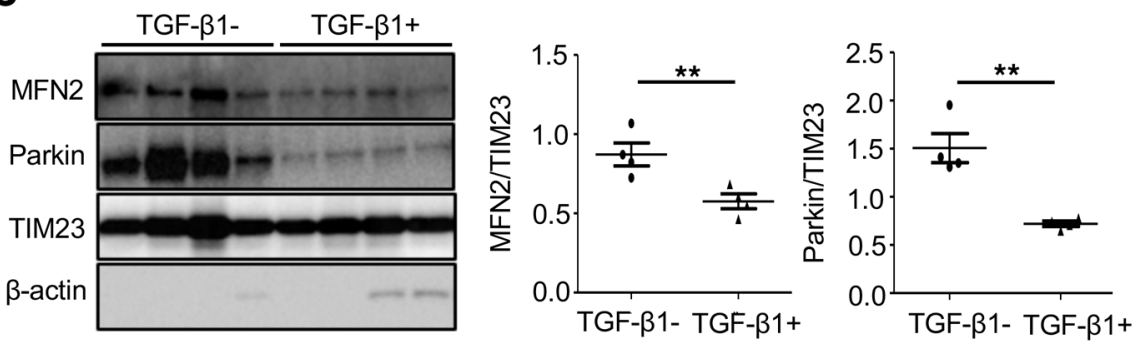

E

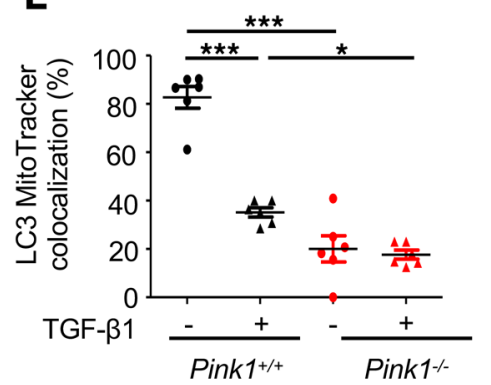

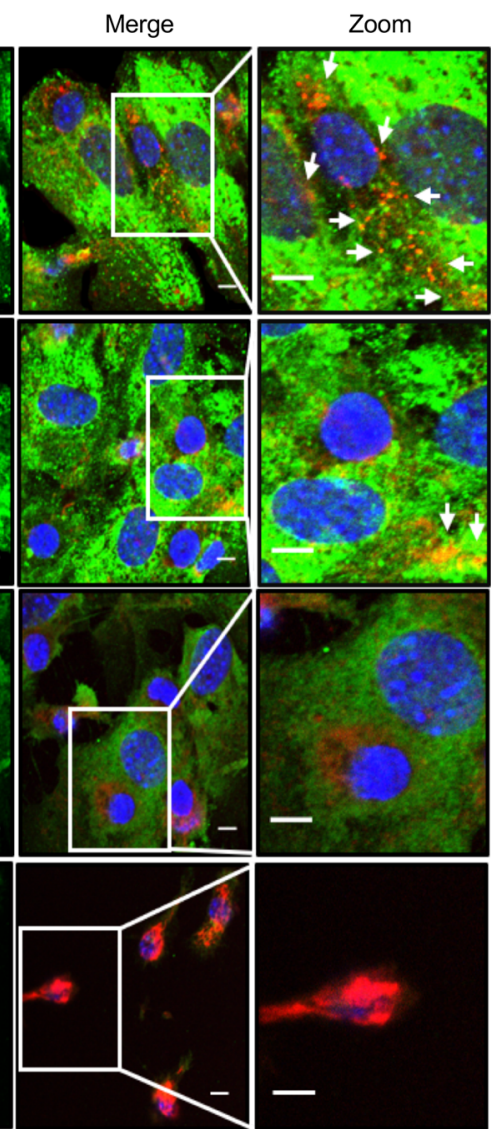

Pink1+/
F

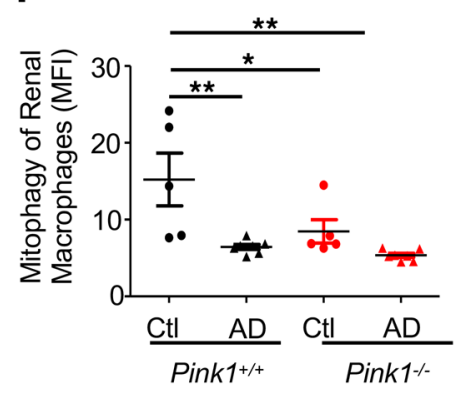

G

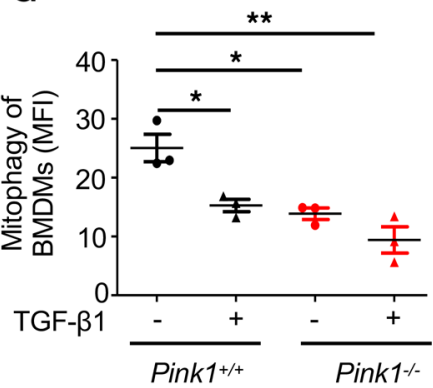

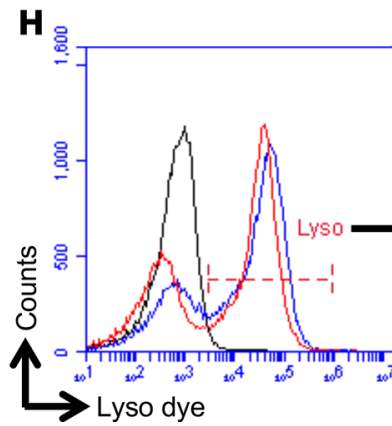

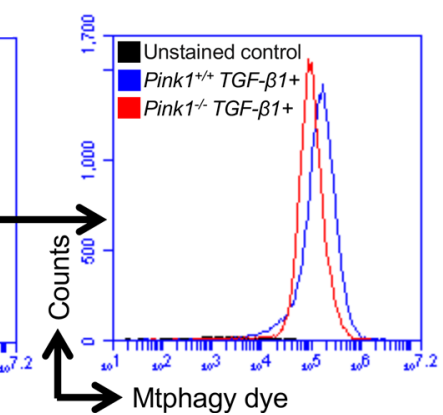

Figure 5. Macrophage PINIK1-mediated mitophagy is suppressed during fibrotic conditions. (A) Western blot and densitometry analysis for PINK1 expression normalized to $\beta$-actin in THP-1-derived human macrophages cultured in the absence (-) or presence ( + ) of TCF- $\beta 1$ (5 ng/mL) for 48 hours ( $n=5$ per group). (B) Western blot and densitometry analysis for MFN2 and Parkin normalized to GAPDH in renal macrophages isolated from WT mice 7 days after sham or UUO surgery ( $n=3$ per group). (C) Western blot and densitometry analysis for MFN2 and Parkin normalized to TIM23 in mitochondrial lysates from BMDMs ( $n=4$ per group) cultured in the absence (-) or presence (+) of TGF- $\beta 1$ ( $5 \mathrm{ng} / \mathrm{mL})$ for 48 hours. $\beta$-Actin (a cytosolic marker) was used to confirm the purity of mitochondrial fractions. (D and E) Representative confocal microscopy images (D) and quantification of mitochondria colocalized with LC3 (E). Pink $1^{+/+}$and Pink $1^{1 /-}$ BMDMs cultured in absence or presence of TCF- $\beta 1$ were analyzed using 
MitoTracker (red) dye, anti-LC3 (green), and Hoechst (blue) dye. White arrows indicate colocalization of LC3 with mitochondria. Over 100 cells from 6 random fields per experimental group, each in triplicate, were analyzed using ImageJ. Scale bar: $10 \mathrm{~mm}$. (F) Mitophagy assessment in Pink $7^{+/+}$and Pink $1^{-1-} \mathrm{F} 4 / 80^{+}$renal macrophages from mice fed with control ( $\mathrm{Ctl}, n=5$ per group) or adenine (AD, $n=7$ per group) diet for 28 days and stained with MitoTracker and LysoTracker dyes using flow cytometry. (G) Mitophagy assessment in Pink $1^{+/+}$and Pink $1^{1-}$ BMDMs ( $n=3$ per group) cultured in the absence or presence of TGF- $\beta 1(5 \mathrm{ng} / \mathrm{mL})$ for 48 hours using flow cytometry. (H) Representative histograms for the detection of mitophagy showing the lyso dye-labeled positive lysosomes gated for Mtphagy dye-stained mitochondria. Data are mean \pm SEM representative of 3 independent experiments and analyzed by Student's unpaired 2-tailed $t$ test (A, B, and $\mathbf{C}$ ) or 1-way ANOVA (E, $\mathbf{F}$, and $\mathbf{G}) .{ }^{*} P<0.05 .{ }^{* *} P<0.01,{ }^{* * *} P<0.001$.

PINK1-dependent recruitment of Parkin to mitochondria has been shown to promote mitophagy $(30,31)$. Thus, we investigated the role of MFN2 in the recruitment of Parkin to the mitochondria of macrophages. We isolated the mitochondrial fractions from the peritoneal macrophages cultured in the presence of DMSO (vehicle control) or carbonyl cyanide 4-(trifluoromethoxy) phenylhydrazone (FCCP) $(5 \mu \mathrm{M} / \mathrm{mL})$ for 2 hours and analyzed for expression of Parkin and mitochondrial marker TOM20. Mitochondrial uncoupler FCCP induced recruitment of Parkin to the depolarized mitochondria, as confirmed in LysM-Cre ${ }^{-/-} M f n 2^{f / f l}$ macrophages (Figure 6D). However, in LysM-Cre ${ }^{+/-} M f n 2^{f / f l}$ macrophages, the FCCP-induced recruitment of Parkin to the mitochondria was considerably lower compared with LysM-Cre $e^{-/-} M f n 2^{f / f l}$ macrophages. These findings suggest that MFN2 may function upstream of Parkin and that, following PINK1-dependent activation of mitophagy, MFN2 promotes the recruitment of Parkin to the mitochondria in macrophages.

PINK1 deficiency impairs macrophage mitochondrial homeostasis during kidney fibrosis. To further examine the role of TGF- $\beta 1-$ mediated suppression of the expression of mitophagy regulators in macrophages, we studied the effects of TGF- $\beta 1$ on macrophage mitochondrial respiration as assessed by Mito Stress test. We show that the macrophage mitochondrial respiration was decreased after treatment with TGF- $\beta 1$. Pink1 $1^{-/-}$BMDMs after TGF- $\beta 1$ treatment displayed significantly lower mitochondrial oxygen consumption rate (OCR), ATP production, spare respiratory capacity, and maximal respiration than $P i n k 1^{+/+} \mathrm{BMDMs}$ (Figure 7A). There were no differences in the rate of nonmitochondrial respiration between Pink1 ${ }^{+/+}$and Pink1 ${ }^{-/-}$BMDMs. TGF- $\beta 1-$ treated Pink $^{-{ }^{--}}$BMDMs exhibited higher production of mitochondrial-derived superoxide ions as measured by MitoSox staining (Figure 7B). These data suggest that TGF- $\beta 1$ negatively affects macrophage mitochondrial homeostasis and that PINK1 deficiency further impairs the macrophage mitochondrial metabolic health.

We next studied the impact of the loss of PINK1-dependent mitophagy on the renal macrophage mitochondrial injury in experimental kidney fibrosis. We used transmission electron microscopy to analyze the ultrastructure of the renal macrophage mitochondria in the kidneys from Pink1 ${ }^{+/+}$and Pink1 $1^{-/-}$mice after 28 days of $\mathrm{AD}$ or control diet. Evidence of mitochondrial swelling in the renal macrophages was observed in Pink $1^{+/+}$mice after AD-induced fibrosis. Renal macrophages from Pink1 $^{-/-}$mice fed with control diet displayed

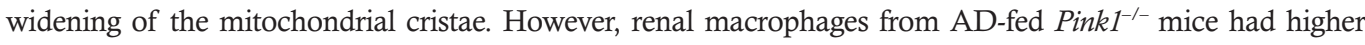
numbers of abnormal mitochondria, with evidence of reduced density of matrix and disorganized cristae, compared with Pink1 ${ }^{+/+}$AD-fed mice (Figure 7C). These data suggest that lack of PINK1-mediated mitophagy-dependent elimination of damaged mitochondria contributes to their accumulation. Collectively, the above findings provide evidence that PINK1 exerts a critical role in maintaining renal macrophage mitochondrial quality control, and its deficiency contributes to macrophage mitochondrial injury during kidney fibrosis.

Mitophagy regulators PINK1, MFN2, and Parkin are repressed in the kidney and in circulating mononuclear cells from patients with $C K D$. Increased macrophage infiltration (32) and mitochondrial impairment (33) have been reported in patients with kidney diseases. Here, we explored the role of mitophagy in human kidney disease. Kidney allograft biopsy samples obtained from CKD patients with histological evidence of interstitial fibrosis and tubular atrophy (IFTA) (Table 1) displayed significantly lower mRNA expression of mitophagy regulators PINK1 (Figure 8A), MFN2 (Figure 8B), and PRKN (Figure 8C), compared with kidney biopsy samples from patients with no $\mathrm{CKD}\left(\mathrm{CKD}^{-}\right)$. Furthermore, circulating levels of chemokine CCL2 were higher in the plasma samples from CKD patients (Figure 8D). CCL2 acts as a ligand for CCR2-expressing macrophages, and increased levels are known to promote macrophage infiltration (34) and progressive tubulointerstitial kidney disease (35). Our findings suggest that, in CKD, mitophagy is suppressed, while increased circulating CCL2 induces macrophage infiltration to promote kidney fibrosis.

Similarly, peripheral blood mononuclear cells (PBMCs) from patients with severe CKD displayed lower expression of MFN2 (Figure 8E) and Parkin (Figure 8F), and higher mitochondrial-derived superoxide production (Figure $8 \mathrm{G}$ ), than patients with mild or moderate CKD (Table 2). Higher mROS levels and 

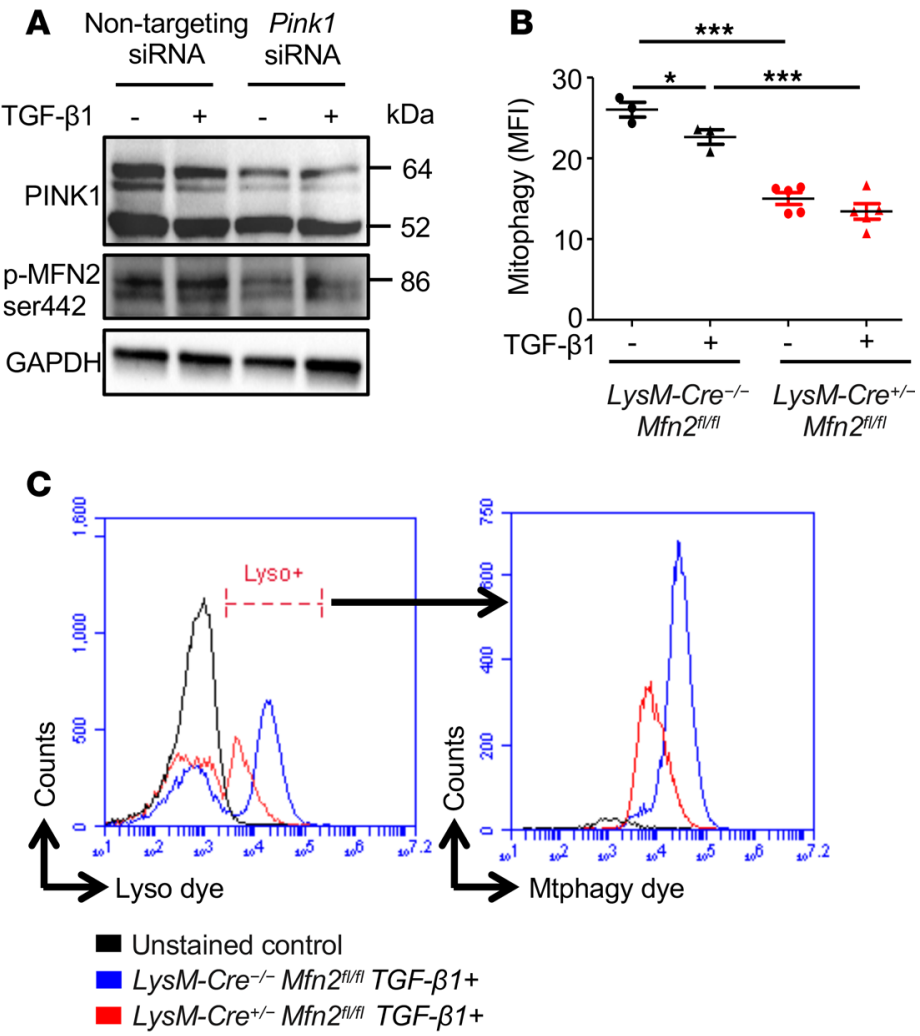

D

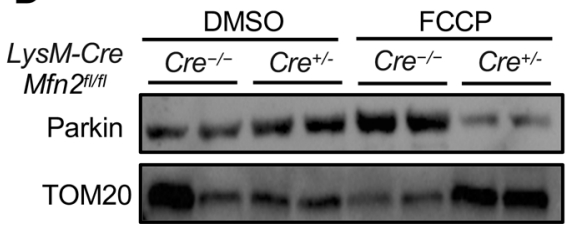

Figure 6. PINIK1 mediates phosphorylation of MFN2 and MFN2 facilitates Parkin recruitment to the macrophage mitochondria. (A) THP-1-derived human macrophages transfected with Pink1 siRNA or nontargeting (NT) control siRNA, cultured in the absence (-) or presence (+) of TCF- $\beta 1$ ( $5 \mathrm{ng} / \mathrm{mL})$ for 48 hours. Western blot for PINK1 (full length, $64 \mathrm{kDa}$; cleaved, $52 \mathrm{kDa}$ ) and phosphorylated MFN2 Serine-442 (86 kDa). (B) Mitophagy measured in LysM-Cre ${ }^{-/-} M f n 2^{f / f l}$ ( $n=3$ per group) and LysM-Cre ${ }^{+/-} M f n 2^{f / / f l}$ BMDMs ( $n=5$ per group) cultured in the absence (-) or presence (+) of TCF- $\beta 1$ ( $5 \mathrm{ng} / \mathrm{mL}$ ) for 48 hours (B). (C) Representative histograms for the assessment of mitophagy showing the Lyso dye-positive events, gated for Mtphagy dyestained mitochondria. The mean fluorescence intensity (MFI) of Mtphagy dye-stained mitochondria colocalized with Lyso dye-labeled lysosomes measured using flow cytometry. (D) Western blot for Parkin and TOM2O in peritoneal macrophages isolated from LysM-Cre ${ }^{-/-} \mathrm{Mfn} 2^{\mathrm{fl} / \mathrm{fl}}$ and LysM-Cre $\mathrm{Cr}^{+/-}$ $M f n 2^{f / / f l}$ cultured in the presence of DMSO (vehicle control) or FCCP $(5 \mu \mathrm{M} / \mathrm{mL})$ for 2 hours. Data are mean \pm SEM and representative of 2 independent experiments. ${ }^{*} P$ $<0.05$, ${ }^{* *} P<0.001$ analyzed by 1 -way ANOVA.

lower expression of mitophagy regulatory proteins in patients with severe kidney disease provide evidence that mitophagy in mononuclear cells decreased with the progression of CKD.

Mitophagy controls human primary renal macrophage-induced fibrotic response. We next isolated the macrophages from human kidney (Supplemental Figure 4B) and investigated the effects of TGF- $\beta 1$ on the expression of mitophagy regulators. TGF- $\beta 1$ treatment of human primary renal macrophages resulted in decreased expression of PINK1, MFN2, and Parkin, along with an increase in the fibrotic response, as assessed by FN and CX3CR1 expression (Figure 8H). In line with our above findings, these data suggest that increased fibrotic response by renal macrophages is associated with decreased expression of mitophagy regulators.

We next determined the effect of mitochondrial division inhibitor 1 (Mdivi1), a mitophagy inhibitor that has also been shown to modulate mROS production through mitochondrial complex I (36). We observed that Mdivi1-treated human renal macrophages showed reduced expression of mitophagy mediators (PINK1, MFN2, and Parkin) (Figure 8I) and lower mitochondrial membrane potential than DMSO-treated (vehicle) cells (Figure 8J). The decrease in the expression of mitophagy mediators was associated with increased expression of TGF- $\beta 1$, CD206, CX3CR1, FN, and $\alpha$-SMA (Figure 8I).

\section{Discussion}

Mitophagy is found to be induced during acute kidney injury $(14,22,37,38)$ and compromised during CKD in animal models $(39,40)$. Mitochondrial dysfunction is also reported in human kidney disease (33). However, the role of macrophage mitophagy in the pathogenesis of kidney fibrosis in progressive CKD is largely unknown. Our findings utilizing 2 different models of kidney fibrosis indicate that the renal expression of mitophagy mediators is downregulated during kidney fibrosis and implicate the relevance in the development of kidney fibrosis. The present study provides the first demonstration to 

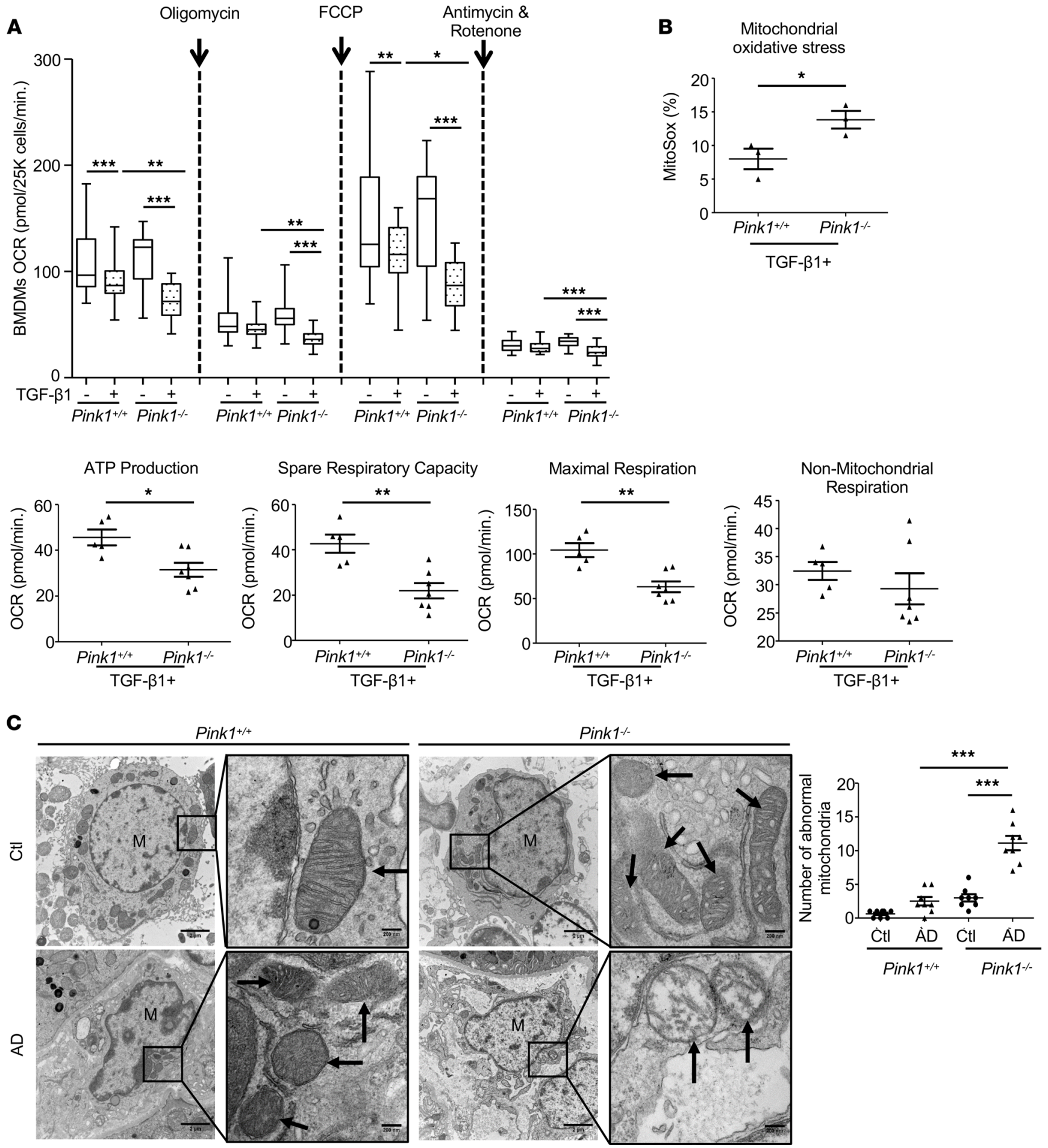

Figure 7. PINK1 mediates macrophage mitochondrial respiration during kidney fibrosis. (A) Pink1+/+ and Pink1 $1^{-/-}$BMDMs were cultured in the absence $(-, n=5$ per group) or presence (+, $n=5$ per group) of TCF- $\beta 1(5 \mathrm{ng} / \mathrm{mL})$ for 24 hours. Box-and-whisker plots displaying the first and the third quartiles, with the line within the box indicating the median value (upper panel). Oxygen consumption rate (OCR) under basal conditions followed by the sequential measurements after addition of oligomycin, FCCP, rotenone, or antimycin A were determined using Mito Stress test. Dot plots show mean \pm SEM. (B) Frequency of mitochondrial-derived superoxide detected in Pink $1^{+/+}$and Pink $1^{-/-}$TGF- $\beta 1$-treated BMDMs ( $n=3$ per group) using MitoSox staining by flow cytometry. (C) Representative transmission electron microscopy (TEM) images displaying renal macrophages (labeled as M; $\times 12,000$ magnification; scale bars: $2 \mu \mathrm{m}$ ) and their mitochondria (pointed by arrow, $\times 80,000$ magnification; scale bars: $200 \mathrm{~nm}$ ) in Pink ${ }^{+/+}$and Pink1 ${ }^{-/-}$mice after 28 days of control (Ctl) or adenine (AD) diet. Abnormal mitochondria counted from 4 (CtI) or $7(\mathrm{AD})$ renal macrophages from $n=3$ mice per group. Data are mean $\pm \mathrm{SEM}$. ${ }^{*} P<0.05$. ${ }^{* *} P<0.01,{ }^{* * *} P<0.001$ analyzed by 1-way ANOVA (A, upper panel, and $\mathbf{C}$ ) or Student's unpaired 2-tailed $t$ test (A, lower panels, and $\mathbf{B}$ ). 
Table 1. Clinical characteristics of adult patients with and without CKD

\begin{tabular}{|c|c|c|c|}
\hline Cohort characteristics & Non-CKD $(n=9)$ & CKD $(n=6)$ & $P$ value \\
\hline Sex, male/female & $5 / 4$ & $2 / 4$ & $0.398^{\mathrm{A}}$ \\
\hline Urine ACR (mg/g) & $50.4(7.9-234.1)$ & $762.8(74.8-1413)$ & 0.009 \\
\hline Serum creatinine (mg/dL) & $1.9(1.6-2.5)$ & $2.3(1.9-3.6)$ & 0.044 \\
\hline BUN (mg/dL) & $26.0(22.5-36.0)$ & $49.0(27.5-63.8)$ & 0.009 \\
\hline Basiliximab & $2 / 9$ & $1 / 6$ & 0.810 \\
\hline ATC & $7 / 9$ & $5 / 6$ & 0.810 \\
\hline \multicolumn{4}{|c|}{ Maintenance Immunotherapies } \\
\hline Tacrolimus & $9 / 9$ & $5 / 6$ & 0.234 \\
\hline MMF & $9 / 9$ & $5 / 6$ & 0.234 \\
\hline
\end{tabular}

GFR, glomerular filtration rate; ACR, albumin/creatinine ratio; BUN, blood urea nitrogen; ATG, anti-thymocyte globulin; MMF, mycophenolate mofetil. Continuous variables are presented as median (interquartile range) and analyzed using unpaired 1-tailed $t$ test. ${ }^{A}$ Proportions analyzed using $\chi^{2}$ test.

our knowledge that macrophage mitophagy is protective against kidney fibrosis via the PINK1/MFN2/ Parkin-mediated pathway.

The paradigm that mitophagy protects against kidney fibrosis by modulating the macrophage-derived fibrotic response is in accordance with evidence provided by previous reports. For example, autophagy regulates the macrophage-derived mitochondrial superoxide production, as both Beclin-1- or LC3-deficient macrophages produce higher superoxide anion radicals $\left(\mathrm{O}_{2}^{-}\right)(41)$. Chemical inhibition of autophagy in macrophages using 3-methyladenine results in increased numbers of damaged mitochondria and the production of mROS (42). In addition, the increase in the ROS levels is associated with enhanced differentiation of alternatively activated/M2 macrophages (43). In the mouse model of diabetic nephropathy, mitochondrial-targeted antioxidant (MitoQ), by scavenging mROS, has been shown to protect against high glucose-induced renal damage and mitochondrial dysfunction (40).

Our conclusion that PINK1-mediated mitophagy modulates macrophage-derived fibrotic response is supported by several compelling observations. First, we show that the renal macrophages from fibrotic kidneys of both $\mathrm{Pink1}^{-/-}$or $\mathrm{Prkn}^{-1-}$ mice display the amplified fibrotic response. Macrophage-specific deletion of $M f n 2$ aggravates kidney fibrosis. Second, the circulating and renal $\mathrm{Ly} 6 \mathrm{C}^{\mathrm{lo}} \mathrm{CD} 11 \mathrm{~b}^{+}$monocytes and renal profibrotic/M2 macrophages are higher, while the Ly6 $\mathrm{C}^{\text {hi }}$ proinflammatory and Ly6 $\mathrm{C}^{\text {int }}$ monocytes are lower during kidney fibrosis in $\mathrm{Pink1}^{-/-}$or $\mathrm{Prkn}^{-/-}$or $\mathrm{LysM}-\mathrm{Cr} \mathrm{f}^{+/-} \mathrm{Mfn} 2^{f / f l}$ mice. Ly6C $\mathrm{C}^{\text {lo }}$ monocytes and profibrotic macrophages contribute to advancing kidney fibrosis $(4,7,25,27)$, while the Ly6C $C^{\text {int }}$ population plays reparative functions during kidney injury (25). Third, both Pink1 ${ }^{-/-}$or $\mathrm{Prkn}^{-1-}$ renal macrophages show higher expression of Gal-3, a M2/profibrotic marker during kidney fibrosis. Fourth, both Pink1 ${ }^{-/-}$or Prkn ${ }^{-/}$BMDMs exhibit enhanced IL-4-induced polarization toward M2 phenotype in the presence of macrophage-CSF (M-CSF) conditioned media. The mechanism through which these mitophagy mediators regulate the macrophage-polarization toward M2 phenotype may be rictor dependent. Loss of Pink1 in BMDMs results in greater mitochondrial-derived superoxide production after TGF- $\beta 1$ treatment. Previous studies have identified critical roles of rictor $(28,29,44)$ and mitochondrial-derived ROS (43) in promoting macrophage differentiation toward M2 phenotype. Mitophagy is also demonstrated to regulate the expression of TGF- $\beta 1 \mathrm{mRNA}$ in alveolar macrophages (45). However, no study has investigated the role of PINK1-mediated mitophagy in macrophage reprogramming, particularly in the context of kidney fibrosis.

The reduced expression of mitophagy regulators in both the renal macrophages from the fibrotic kidney and the mitochondrial fractions from TGF- $\beta 1$-treated BMDMs suggest that macrophage mitophagy is compromised during kidney fibrosis. We demonstrate that the numbers of abnormal mitochondria in 
A

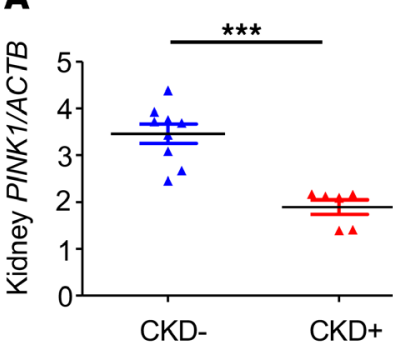

E

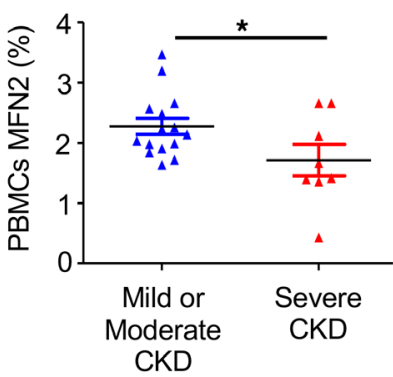

H

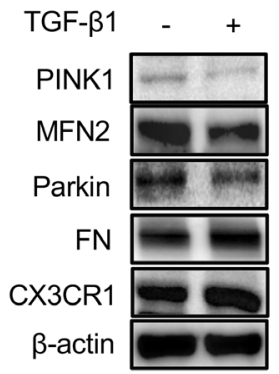

B

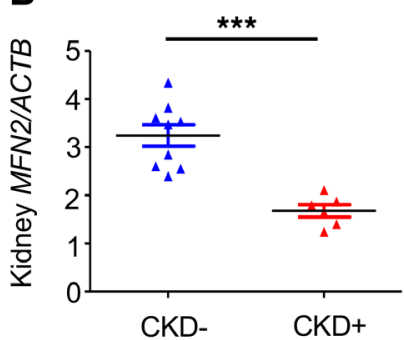

F

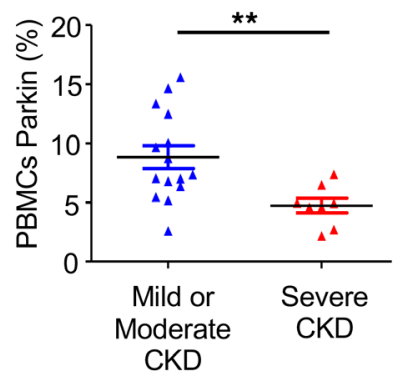

I

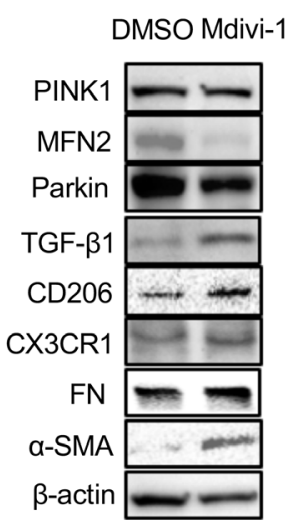

C
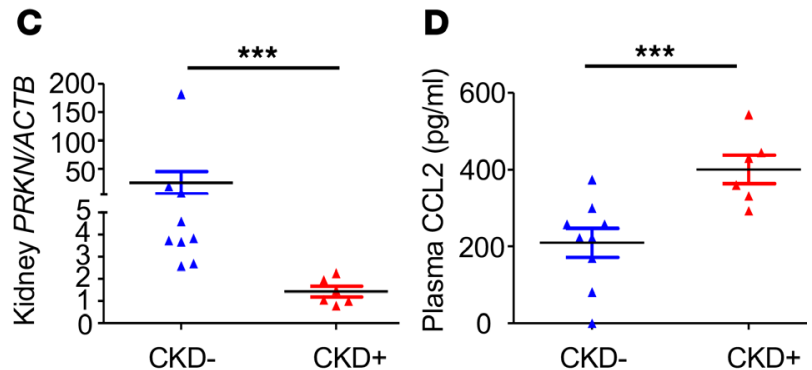

G

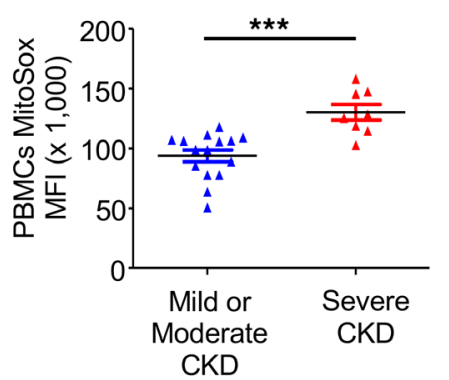

J

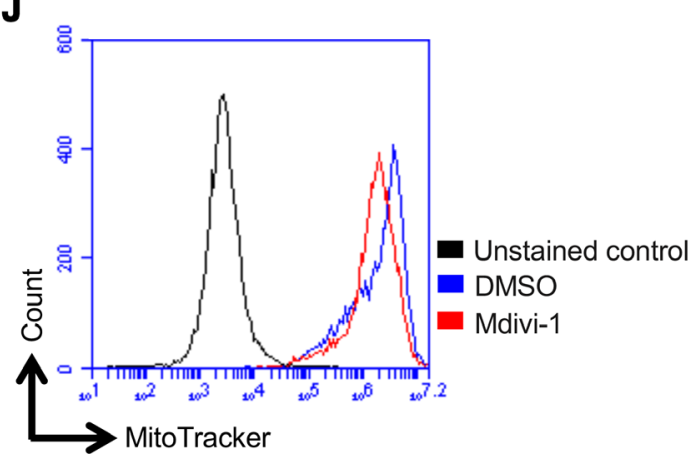

Figure 8. Mitophagy is compromised in patients with CKD and in TGF- $\beta$ 1-treated human renal macrophages. (A-C) Relative mRNA expression levels of PINK1 (A), MFN2 (B), and PRKN (C) normalized with $\beta$-actin (ACTB) were determined by TaqMan qPCR in kidney biopsy from patients with CKD (CKD ${ }^{+}, n=$ 6) and patients without CKD (CKD-, $n=9$ ) and analyzed using Mann-Whitney $U$ test. (D) Plasma CCL2 levels in patients with (CKD+, $n=6)$ or without CKD (CKD-, $n=9$ ) were determined by ELISA and analyzed using Mann-Whitney $U$ test. (E-G) Frequency of MFN2 (E) and Parkin (F) and median fluorescence intensity (MFI) of MitoSox dye (G) in peripheral blood mononuclear cells (PBMCs) from patients with severe CKD $(n=8)$ vs. mild or moderate CKD ( $n$ $=15$ ) were determined by flow cytometry and analyzed using student's unpaired 2-tailed $t$ test. (H) Western blot for the expression of PINK1, MFN2, Parkin, fibronectin (FN), CX3CR1, and $\beta$-actin in human primary renal macrophages cultured in the absence (-) or presence (+) of TGF- $\beta 1$ (5 ng/mL) for 24 hours. (I) Western blot for the expression of PINK1, MFN2, Parkin, TCF- $\beta 1$, CD206, CX3CR1, FN, $\alpha$-SMA, and $\beta$-actin in human primary renal macrophages cultured in the presence of DMSO (vehicle control) or Mdivi-1 (50 $\mu \mathrm{M})$ for 3 hours. (J) Mitochondrial membrane potential in human primary renal macrophages treated with DMSO (vehicle control) or Mdivi-1 $(50 \mu \mathrm{M})$ for 3 hours detected by staining with MitoTracker dye through flow cytometry. The histogram was gated from forward scatter (FSC) vs. side scatter (SSC) parent population. Data are mean $\pm \mathrm{SEM},{ }^{*} P<0.05,{ }^{* *} P<0.01,{ }^{* * *} P<0.001$.

Pink $1^{-1-}$ renal macrophages are increased in kidney fibrosis induced after 28 days of $\mathrm{AD}$, suggesting the role of PINK1-mediated mitophagy in macrophage mitochondrial quality control. We show that, after AD-induced kidney fibrosis and in response to TGF- $\beta 1$, mitophagy in renal macrophages and BMDMs respectively decreases. The loss of Pink1 was associated with reduced mitophagy in macrophages. Our data also show that the expression of mitophagy regulatory proteins in the mitochondria of BMDMs decreases after TGF- $\beta 1$ treatment. The decline in OCR, ATP production, and maximal respiration by BMDMs in response to TGF- $\beta 1$ treatment further confirms that macrophage-mitochondrial respiration is impacted during fibrotic conditions. Our findings confirm that PINK1 exerts critical function in maintaining macrophage-mitochondrial homeostasis. Accordingly, TGF- $\beta 1$-treated Pink1 $1^{-1-}$ BMDMs display 
Table 2. Clinical characteristics of pediatric patients with CKD

\begin{tabular}{|c|c|c|c|}
\hline Patient characterstics & $\begin{array}{c}\text { Mild or moderate CKD } \\
{\left[\mathrm{GFR} \geq 30 \mathrm{~mL} / \mathrm{min} / 1.73 \mathrm{~m}^{2}\right]} \\
(n=15)\end{array}$ & $\begin{array}{c}\text { Severe CKD } \\
{\left[\mathrm{GFR}<30 \mathrm{~mL} / \mathrm{min} / 1.73 \mathrm{~m}^{2}\right]} \\
(n=8)\end{array}$ & P value \\
\hline Age, years & $14.0(9.0-15.2)$ & $15.9(12.3-17.6)$ & 0.088 \\
\hline Sex, male/female & $10 / 5$ & $2 / 6$ & $0.057^{A}$ \\
\hline GFR (mL/min/1.73 $\left.\mathrm{m}^{2}\right)$ & $48.8(38.0-74.6)$ & $13.9(6.1-25.8)$ & $<0.0001$ \\
\hline Urine ACR $(\mathrm{mg} / \mathrm{g})^{\mathrm{B}}$ & $32(5.2-97)$ & $706.8(58.0-6649.0)$ & 0.026 \\
\hline Serum creatinine (mg/dL) & $1.3(0.83-1.65)$ & $4.4(2.0-10.7)$ & 0.001 \\
\hline BUN (mg/dL) & $25.0(18.0-30.0)$ & $61.5(53.5-78.5)$ & $<0.0001$ \\
\hline
\end{tabular}

GFR, glomerular filtration rate; ACR, microalbumin/creatinine ratio; BUN, blood urea nitrogen. ${ }^{\mathrm{A} P r o p o r t i o n s}$ analyzed by chi-square test. ${ }^{\mathrm{B}} \mathrm{Available}$ on $n$ $=11$ (moderate or mild CKD) and $n=4$ (severe CKD) patients and compared using unpaired 1-tailed $t$ test. Values are expressed as median (interquartile range) and compared using unpaired 2-tailed $t$ test.

lower mitochondrial respiration and higher mitochondrial-derived superoxide production. The mitophagy and mitochondrial respiration are highly interconnected processes. The damaged mitochondria with decreasing membrane potential are usually removed through mitophagy (15). Accumulation of dysfunctional mitochondria due to defective mitophagy in the absence of PINK1 exaggerates mitochondrialderived oxidative stress that may negatively regulate mitochondrial respiration.

The understanding of the molecular regulation of mitophagy in macrophages is not well established. We show that PINK1 functions upstream of MFN2, as the phosphorylation of MFN2 at Ser-442 decreases after PINK1-knockdown in human macrophages. Our observations indicate that the MFN2 bridges between PINK1 and Parkin and facilitates the import of cytosolic Parkin to the depolarized mitochondria, as evidenced by $\mathrm{LysM}-\mathrm{Cr} \mathrm{t}^{+/-} \mathrm{Mfn} 2^{f / f l} \mathrm{BMDMs}$ displaying significantly lower mitophagy with or without TGF- $\beta 1$ treatment. Moreover, the FCCP-induced recruitment of Parkin on the depolarized mitochondria was lower in the absence of MFN2. These findings suggest that the mitophagy in macrophages involves MFN2-dependent recruitment of cytosolic Parkin to the damaged mitochondria. Our findings on the molecular regulation of mitophagy in macrophages support the published studies on the mechanism of mitophagy in other cells $(20,46)$. However, TGF- $\beta 1$-induced decrease in the recruitment of mitophagy regulatory proteins on the mitochondria of macrophages has not been described before, to our knowledge.

Our findings in human primary renal macrophages and CKD patients strongly corroborate our experimental observations from animal models. Our studies confirm that the mitophagy in the kidney and mononuclear cells was impaired, while the circulating levels of chemokine CCL2, which promotes macrophage recruitment into the kidney, was higher in patients with severe CKD. These findings on macrophages and mitophagy are in line with the earlier published studies performed in animal models $(2,4,22,39,40)$ and collectively highlight the cytoprotective role of mitophagy against macrophage-derived progression of kidney fibrosis.

Our investigations using primary human renal macrophages also confirm that PINK1/Parkin-mediated mitophagy regulates macrophage-induced fibrotic response. This is based on our findings that (a) the expression of downstream regulators of PINK1-dependent mitophagy decreases after a TGF- $\beta 1$-induced increase in the fibrotic response and (b) the increase in the expression of fibrotic markers in Mdivi1-treated macrophages is associated with a decrease in the expression of mitophagy regulators. However, further research is required to determine the PINK1/Parkin-dependent mechanism of regulation of macrophage polarization toward M2 phenotype through mROS or rictor.

In conclusion, our present studies provide a mechanistic understanding of the role of PINK1/ MFN2/Parkin-mediated macrophage mitophagy and its role in the context of kidney fibrosis. Our findings indicate that the mitophagy regulators are downregulated in experimental animal models of kidney fibrosis, in renal macrophages from fibrotic kidney, in TGF- $\beta 1$-treated primary mouse BMDMs and human renal macrophages, and in the human CKD kidney. The deficiency of mitophagy regulators contributes to mitochondrial dysfunction, impaired mitophagy, and elevated levels of mROS and rictor expression, both of which promote macrophage reprogramming toward profibrotic/M2 phenotype (Figure 9). Therefore, it may be prudent to investigate the potential of therapeutically targeting 


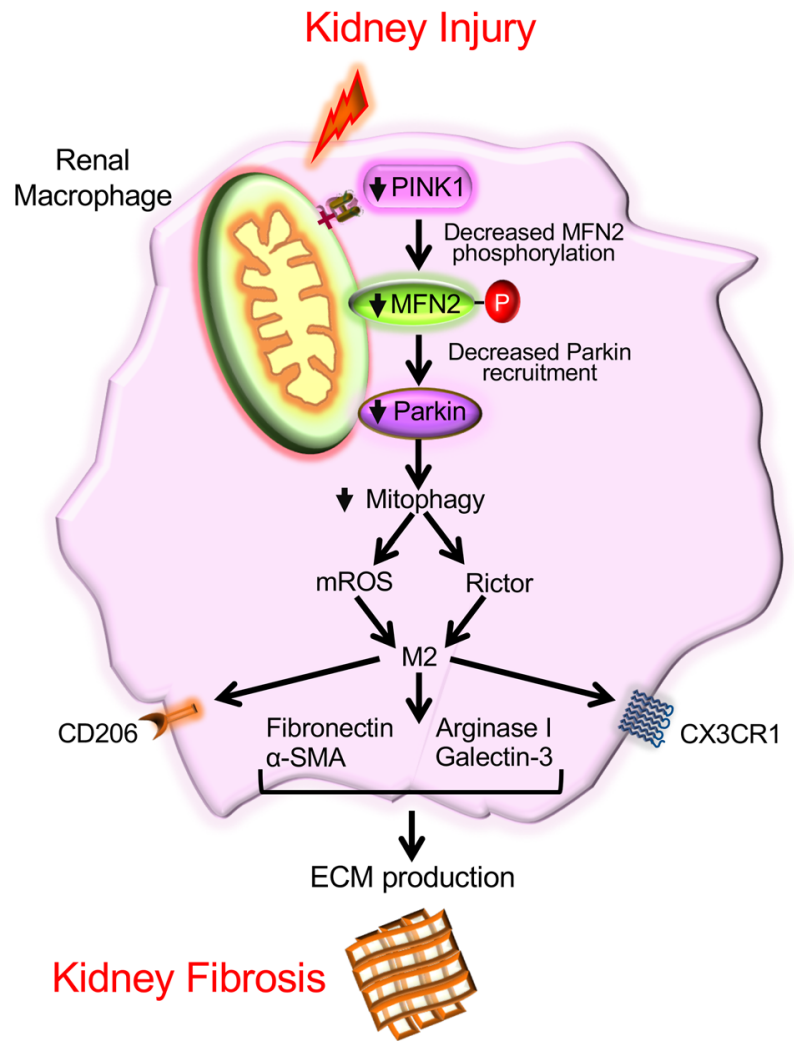

Figure 9. Proposed mechanism of regulation of macrophage-derived fibrotic response by mitophagy. Kidney injury by suppressing the expression of mitophagy regulators (PINK1, MFN2, and Parkin) contributes to mitochondrial dysfunction in renal macrophages. The deficiency of PINK1 causes a reduction in the mitofusin-2 (MFN2) phosphorylation at Serine-442. The loss of MFN2 results in lower recruitment of Parkin to the mitochondria and defective mitophagy in macrophages. The failure of mitophagy causes accumulation of abnormal mitochondria, increase in mROS production, and expression of rictor in macrophages. mROS and rictor both promote the differentiation of macrophages toward profibrotic/M2 phenotype. The increase in the frequency of profibrotic/M2 macrophages results in higher extracellular matrix (ECM) production and progression of kidney fibrosis.

the negative regulators of mitophagy to modulate the macrophage-derived progression of kidney fibrosis and protect against the advancement of CKD.

\section{Methods}

Mice. Pink1 ${ }^{-1-}$ and $\mathrm{Prkn}^{-1-}$ mice were provided by Jie Shen (Center for Neurologic Diseases, Brigham and Women's Hospital, Harvard Medical School, Boston, Massachusetts, USA). C57BL/6 mice were purchased from the Jackson Laboratory. Mfn2 $2^{\text {loxplloxp }}$ (stock no. 029902-UCD) mice were generated by David C. Chan (California Institute of Technology, Pasadena, California, USA) and were purchased from Mutant Mouse Resource \& Research Center. The myeloid-specific Mfn2-deficient strain was generated by crossing the $M f n 2^{\operatorname{loxp} / l o x p}\left(M f n 2^{f / f}\right)$ line to a strain expressing Cre under the control of the endogenous promoter of LysM (LysM-Cre strain; stock no. 004781, the Jackson Laboratory). These myeloid-specific $M f n 2$-deficient mice are herein referred to as $L y s M-C r e^{+/-} M f n 2^{l / f l}$. The $L y s M-C r e^{-/-} M f n 2^{l / f l}$ mice were used as corresponding controls.

UUO model of kidney fibrosis. UUO surgery was performed as previously described (47). Briefly, mice (3-5 per group) were anesthetized, and ureteral obstruction surgery was carried out by double ligation of the left ureter using 4-0 silk suture via a left flank incision. Sham-operated mice had their ureters exposed but not ligated. The kidneys were harvested on day 7 for histology, Western blotting, and flow cytometry. The blood was collected for flow cytometry.

AD-induced model of kidney fibrosis. Mice were fed with $0.2 \%$ of $\mathrm{AD}$ or control diet (Envigo; TD150071) as previously described (48). The kidneys were collected at the end of the study on day 14 or day 28 for Western blotting, hydroxyproline assay, and electron microscopy. Whole blood samples were collected for chemokine analysis.

Cell culture and transfection. For the isolation of BMDMs, BM cell suspension was prepared through flushing femurs and tibias, and it was cultured in the presence of M-CSF $(10 \mathrm{ng} / \mathrm{mL}$; BioLegend) for 7 days (49). The polarization of BMDMs toward M1 macrophages was performed using recombinant IFN- $\gamma$ (rIFN- $\gamma ; 10 \mathrm{ng} / \mathrm{mL}$; BioLegend), LPS (100 ng/mL; Invivogen), and granulocyte M-CSF (GM-CSF; $25 \mathrm{ng} / \mathrm{mL}$; Stemcell Technologies). For M2 macrophages, BMDMs were cultured in the presence of rIL-4 (10 ng/mL; BioLegend) and M-CSF ( $25 \mathrm{ng} / \mathrm{mL}$; BioLegend) for 24 hours. 
Peritoneal macrophages were isolated by recovering peritoneal lavage (49), allowed to adhere to the sterile petri dishes for 3 hours, and subsequently subjected to treatment with FCCP ( $5 \mu \mathrm{M} / \mathrm{mL}$; Abcam) or DMSO (vehicle control) for 2 hours.

The macrophages from a human monocytic THP-1 cell line (ATCC TIB-202) were derived by incubating in the presence of phorbol 12-myristate 13-acetate (PMA; $10 \mathrm{ng} / \mathrm{mL}$; MilliporeSigma) for 3 days. Human SMARTpool on-target plus Pink1-specific (L-004030-00-0005) or nontargeting (D-001810-1005) siRNAs were purchased from Dharmacon (GE Healthcare). THP-1-derived macrophages were transfected with a pool of either Pink1-specific or nontargeting siRNAs using transfection reagent (Lipofectamine RNAimax; Thermo Fisher Scientific) followed by treatment with TGF- $\beta 1$ ( $5 \mathrm{ng} / \mathrm{mL}$; R\&D Systems) for 24 hours.

Isolation of renal macrophages. The macrophages from mouse and human kidneys were isolated using Ficoll-Hypaque (Thermo Fisher Scientific) density gradient centrifugation followed by magnetic-activated cell sorting (MACS) separation protocol (Supplemental Figure 4). Kidney tissue was minced into 1-2 $\mathrm{mm}^{3}$ pieces and digested using collagenase type I ( $2 \mathrm{mg} / \mathrm{mL}$; Thermo Fisher Scientific). The single-cell suspensions obtained after digestion were subsequently incubated with ACK lysing buffer (Thermo Fisher Scientific) for the lysis of RBCs, followed by isolation of renal mononuclear cells (RMNCs) by Ficoll-Hypaque density gradient centrifugation method (50). The macrophages from mouse RMNCs were sorted using anti-CD115 biotin-conjugated antibody and anti-biotin microbeads (Miltenyi Biotec) using MACS cell separation protocol for CD115 positive selection (Supplemental Figure 4A and ref. 28). The macrophages from human RMNCs were sorted using PE-conjugated anti-CD68 antibody and anti-PE ultrapure microbeads (Miltenyi Biotec) using MACS positive cell selection protocol (Supplemental Figure 4B).

PBMC isolation. PBMCs were isolated from whole blood using the Ficoll-Paque (GE Healthcare) density gradient centrifugation method as previously described (50). Blood samples were first diluted in PBS in 1:1 and layered on the surface of Ficoll-Paque and centrifuged at $670 \mathrm{~g}$ for 20 minutes at $20^{\circ} \mathrm{C}$ with no breaks. The mononuclear cells were carefully harvested from the interface of diluted plasma and Ficoll. RBCs were lysed using ACK lysing buffer (Thermo Fisher Scientific). Cells were washed and stored in cryopreservation media containing 50\% FBS (Invitrogen) and 10\% DMSO (Sigma-Aldrich) in RPMI (Thermo Fisher Scientific).

Flow cytometry. The surface and intracellular staining were performed on renal single-cell suspensions, BMDMs, or peritoneal macrophages. The nonspecific sites were blocked by using anti-CD16 and anti-CD32 antibodies (BioLegend, 101302) by incubating cells for 15 minutes at $4^{\circ} \mathrm{C}$. The following antibodies were used for staining: anti-CD45 (BioLegend, clone 30-F11); anti-F4/80 (clone BM8), anti-CD11b (clone M1/70), anti-CD206 (clone MR6F3), and anti-Ly6C (clone HK1.4) (Thermo Fisher Scientific); anti-Gal-3 (BioLegend, clone M3/38); anti-latency associated peptide (LAP or TGF- $\beta 1$ ) (BioLegend, clone TW7-16B4); anti-MFN2 (Abcam, clone 6A8); and anti-Parkin (Cell Signaling Technology, catalog 3132S) antibodies. The fixation permeabilization treatment was performed before staining for an intracellular marker using fix/perm buffer (Thermo Fisher Scientific) for 15 minutes at $4^{\circ} \mathrm{C}$. The secondary fluorochrome-conjugated antibodies used were anti-rabbit Alexa Fluor 488 and anti-mouse Alexa Fluor 647 (Thermo Fisher Scientific; catalogs A-11001 and A-21244, respectively). The stained cells were washed twice with FACS buffer (PBS with $2 \%$ FBS; Invitrogen) and fixed using $2 \%$ paraformaldehyde (Santa Cruz Biotechnology Inc.). The cells were captured using BD Accuri C6 flow cytometer, and the data were analyzed using C6 analysis (BD Biosciences) or FlowJo version 10.8 (Tree Star Inc.) software.

Measurement of mitophagy, membrane potential, and superoxide levels by flow cytometry. The mitophagy in renal single-cell suspensions was determined through flow cytometry staining for MitoTracker and LysoTracker dyes and anti-F4/80 antibody (Thermo Fisher Scientific, clone BM8) as per the manufacturer's protocol. The mitophagy in BMDMs was detected according to the assay protocol (mitophagy detection kit, Dojindo) using flow cytometry $(51,52)$. BMDMs were incubated with Mtphagy dye (100 $\mathrm{nmol} / \mathrm{L})$ at $37^{\circ} \mathrm{C}$ for 1 hour with $5 \% \mathrm{CO}_{2}$ and $95 \%$ humidity, followed by treatment with TGF- $\beta 1$ (5 ng/ $\mathrm{mL}$ ) for 48 hours at $37^{\circ} \mathrm{C}$ in serum-free M-CSF (BioLegend) conditioned media. The cells were then washed and incubated with Lyso dye $(1 \mu \mathrm{mol} / \mathrm{L})$ for 1 hour. The mean fluorescence intensity (MFI) or the counts of the double-positive signal for the Mtphagy dye-stained mitochondria colocalized with Lyso dye-labeled lysosomes were measured using flow cytometry (Accuri C6, BD Biosciences). 
The mitochondrial membrane potential was determined by calculating MFI or positive signal for MitoTracker red dye-stained (50 nM, Thermo Fisher Scientific) cells. The levels of mitochondrial-derived superoxide or mROS were detected by MitoSox staining ( $5 \mu \mathrm{M}$; Thermo Fisher Scientific). The cells were captured using BD Accuri C6 flow cytometer.

Measurement of OCR and ATP production. Mitochondrial respiration was determined using Mito Stress test by Seahorse XF96 Extracellular Flux analyzer (Agilent Technologies) according to the manufacturer's protocol. Briefly, $2.5 \times 10^{4}$ BMDMs were counted using hemocytometer, seeded per well in 96-well Seahorse plate, and incubated overnight in serum-free M-CSF conditioned media, followed by treatment with TGF- $\beta 1(5 \mathrm{ng} / \mathrm{mL})$ for 24 hours at $37^{\circ} \mathrm{C}$ in the $\mathrm{CO}_{2}$ incubator. One hour before the measurement of mitochondrial respiration, the BMDMs were washed, and the media was replaced with $175 \mu \mathrm{L}$ of Seahorse XF RPMI media (without phenol red; Agilent Technologies) supplemented with $1 \mathrm{mM}$ of sodium pyruvate and $2 \mathrm{mM}$ of glucose solution (Sigma-Aldrich) for 1 hour at $37^{\circ} \mathrm{C}$ in the $\mathrm{CO}_{2}$ free incubator. The measurement for OCR on Seahorse analyzer was performed for each well: 3 measurements at the basal level, 3 after automated injection of oligomycin $(1 \mu \mathrm{M})$ FCCP $(1 \mu \mathrm{M})$, and finally, 3 after injections of both rotenone $(1 \mu \mathrm{M})$ and antimycin $\mathrm{A}(1 \mu \mathrm{M})$. The cell counts were confirmed using CyQUANT kit (Thermo Fisher Scientific). The results were analyzed using XF Wave software (Agilent Technologies).

ELISA. The levels of CCL2 in the mouse plasma (LSBio, LS-F271-1) and human plasma (R\&D Systems, DCP00) samples were quantitated using sandwich ELISA by following manufacturer's instructions.

Transmission electron microscopy. Kidney tissues were fixed with $2.5 \%$ glutaraldehyde (Sigma-Aldrich), $4 \%$ paraformaldehyde (Santa Cruz Biotechnology Inc.), and 0.02\% picric acid in $0.1 \mathrm{M}$ sodium cacodylate buffer (Sigma-Aldrich) at $\mathrm{pH} 7.2$ for overnight at $4^{\circ} \mathrm{C}$. Tissues were washed twice with $0.1 \mathrm{M}$ sodium cacodylate buffer. Following a secondary fixation in $1 \%$ osmium tetroxide and $1.5 \%$ potassium ferrocyanide (Sigma-Aldrich), the samples were dehydrated through a series of graded ethanol (Thermo Fisher Scientific) and embedded in an EPON analog resin (Sigma-Aldrich). Ultrathin sections were cut using Accu-Edge high-profile blades (Sakura, 4685) on a Microm HM 355S (Thermo Fisher Scientific). Sections were placed on the copper grids, contrasted with lead citrate, and captured on the electron microscope (JEM 1400, JEOL). Images were recorded with the associated digital camera (Veleta $2 \mathrm{~K}$, Olympus-SIS). The number of abnormal mitochondria in renal macrophages ( 3 and 10 cells for control diet and AD, respectively) were calculated using ImageJ software (NIH).

TaqMan quantitative PCR. Total RNA was extracted from human kidney tissue using RNeasy mini kit (Qiagen, 74104) according to the manufacturer's instructions. The mRNA was converted into cDNA using SuperScript III reverse transcriptase kit (Invitrogen) and subjected to quantitative PCR (qPCR). The relative expression levels of PINK1 (Hs00260868), MFN2 (Hs00208382), and PRKN (Hs01038318) mRNA were determined using the quantitative TaqMan qPCR method. qPCR was performed in the ABI PRISM 7500 Real-Time PCR System (Applied Biosystems). The data were normalized to a housekeeping gene $A C T B$ (Hs01060665). The gene expression assay reagents were procured from Thermo Fisher Scientific.

Western blot, IHC, and immunofluorescence assay. The mouse kidney tissue and BMDMs were harvested in tissue extraction (Thermo Fisher Scientific, 78510) and mammalian protein extraction (Thermo Fisher Scientific, 78501) buffers, respectively, containing protease inhibitor cocktail (Thermo Fisher Scientific, 78441). Mitochondrial fractions were isolated using the mitochondrial isolation kit (Biovision, K265-25). Protein concentrations were determined using the BCA assay (Thermo Fisher Scientific, 23225). The membranes were subjected to Western blot analysis with the antibodies against FN (Abcam, 2413), Col-I (Abcam, 21286), TGF- $\beta 1$ (Cell Signaling Technology, 3711S), CD206 or mannose receptor-1 (Abcam, 64693), Arg-I (Santa Cruz Biotechnology Inc., 271430), Gal-3 (Santa Cruz Biotechnology Inc., 32790), MFN2 (Abcam, 56889), phospho-MFN2 (Ser-442) (MilliporeSigma, ABC963), PINK1 (Santa Cruz Biotechnology Inc., 517353), Parkin (Cell Signaling Technology, 2132S), LC3 (Sigma-Aldrich, L7543), TIM23 (BD Biosciences, 611223) and human or mouse $\beta$-actin (Cell Signaling Technology, 2132S). Chemiluminescent bands were visualized using enhanced chemiluminescence solution (Thermo Fisher Scientific). Quantification of Western blots was performed using ImageJ software version $1.46(\mathrm{NIH})$.

For IHC, kidney tissues from mice after UUO or Sham surgery or from the mice fed with AD or control diet were fixed overnight in $4 \%$ paraformaldehyde. Sections were dehydrated and embedded in paraffin blocks. Sections $(4-\mu \mathrm{m})$ were collected on Superfrost Plus positively charged microscope slides (Thermo Fisher Scientific), deparaffinized, and stained with Masson's trichrome. 
Immunofluorescence (IF) staining for confocal microscopy on BMDMs was performed using antiLC3 antibody (Sigma-Aldrich, L7543) and MitoTracker Deep Red (Invitrogen, M22426) and Hoechst blue (Thermo Scientific, 62249) dyes. The images were captured on Zeiss LSM 880 confocal microscope at $\times 40$ magnification with a zoom factor of 1.6. The colocalization of LC3 (green) on MitoTracker (red) dye was quantified using ImageJ software (NIH).

Statistics. The statistical analysis was performed on statistical software (GraphPad Prism version 5.0). Data were analyzed using 1-way ANOVA followed by Bonferroni post hoc or Newman-Keuls post hoc test. To compare 2 groups, data were analyzed by Student's unpaired $t$ test or Mann-Whitney $U$ test. The proportions were analyzed using $\chi^{2}$ test.

Study approvals. All animal experiments were performed under protocols approved by the IACUC of Weill Cornell Medicine. Human blood and kidney biopsy samples were obtained under the approved protocols by the IRB registry study at Weill Cornell Medicine. Human kidneys used for macrophage isolation were obtained from the New York organ procurement organization (OPO). These deidentified kidneys, from deceased organ donors, were rejected for clinical transplantation and are not considered as "human subject research," as per the standard NIH definition. Therefore, no IRB approval of the protocol for the kidneys tissue used for the macrophage isolation was required.

\section{Author contributions}

DB and MEC designed the project. DB planned and executed all experiments. KPC, KN, EP, MCR, and LKT contributed to the experimental plan. DB, AMKC, and MEC analyzed the data and wrote the manuscript. OMA and TM contributed human clinical samples. MEC supervised the project.

\section{Acknowledgments}

This work was supported in part by the NIH grants R01 HL133801 and R01 HL132198 to MEC and AMKC.

Address correspondence to: Mary E. Choi, Division of Nephrology and Hypertension, Joan and Sanford I. Weill Department of Medicine, Weill Cornell Medicine, 525 East 68th Street, Box 3, New York, New York 10065, USA. Phone: 646.962.2605; Email: mechoi@med.cornell.edu. Or to: Augustine M. K. Choi, Division of Pulmonary and Critical Care Medicine, Joan and Sanford I. Weill Department of Medicine, Weill Cornell Medicine, 1300 York Avenue, Suite F-113 Box 83, New York, New York 10065, USA. Phone: 212.746.6005; Email: amc2056@med.cornell.edu.

1. Lee SY, Kim SI, Choi ME. Therapeutic targets for treating fibrotic kidney diseases. Transl Res. 2015;165(4):512-530

2. Nikolic-Paterson DJ, Wang S, Lan HY. Macrophages promote renal fibrosis through direct and indirect mechanisms. Kidney Int Suppl (2011). 2014;4(1):34-38.

3. Lee S, et al. Distinct macrophage phenotypes contribute to kidney injury and repair. J Am Soc Nephrol. 2011;22(2):317-326.

4. Peng X, Zhang J, Xiao Z, Dong Y, Du J. CX3CL1-CX3CR1 Interaction Increases the Population of Ly6C(-)CX3CR1(hi) Macrophages Contributing to Unilateral Ureteral Obstruction-Induced Fibrosis. J Immunol. 2015;195(6):2797-2805.

5. Lin SL, Castaño AP, Nowlin BT, Lupher ML, Duffield JS. Bone marrow Ly6Chigh monocytes are selectively recruited to injured kidney and differentiate into functionally distinct populations. J Immunol. 2009;183(10):6733-6743.

6. Anders HJ, Ryu M. Renal microenvironments and macrophage phenotypes determine progression or resolution of renal inflammation and fibrosis. Kidney Int. 2011;80(9):915-925.

7. Tang PM, Nikolic-Paterson DJ, Lan HY. Macrophages: versatile players in renal inflammation and fibrosis. Nat Rev Nephrol. 2019;15(3):144-158.

8. Wang S, et al. TGF- $\beta /$ Smad3 signalling regulates the transition of bone marrow-derived macrophages into myofibroblasts during tissue fibrosis. Oncotarget. 2016;7(8):8809-8822.

9. Chung S, et al. TGF- $\beta$ promotes fibrosis after severe acute kidney injury by enhancing renal macrophage infiltration. JCI Insight. 2018;3(21):123563.

10. Huen SC, Moeckel GW, Cantley LG. Macrophage-specific deletion of transforming growth factor- $\beta 1$ does not prevent renal fibrosis after severe ischemia-reperfusion or obstructive injury. Am J Physiol Renal Physiol. 2013;305(4):F477-F484.

11. Tan RJ, Liu Y. Macrophage-derived TGF- $\beta$ in renal fibrosis: not a macro-impact after all. Am J Physiol Renal Physiol. 2013;305(6):F821-F822.

12. Sorrentino V, Menzies KJ, Auwerx J. Repairing Mitochondrial Dysfunction in Disease. Annu Rev Pharmacol Toxicol. 2018;58:353-389

13. Bhargava P, Schnellmann RG. Mitochondrial energetics in the kidney. Nat Rev Nephrol. 2017;13(10):629-646.

14. Kaushal GP, Shah SV. Autophagy in acute kidney injury. Kidney Int. 2016;89(4):779-791.

15. Youle RJ, Narendra DP. Mechanisms of mitophagy. Nat Rev Mol Cell Biol. 2011;12(1):9-14

16. Williams JA, Ding WX. Mechanisms, pathophysiological roles and methods for analyzing mitophagy - recent insights. Biol 
Chem. 2018;399(2):147-178.

17. Bingol B, Sheng M. Mechanisms of mitophagy: PINK1, Parkin, USP30 and beyond. Free Radic Biol Med. 2016;100:210-222.

18. Ding WX, Yin XM. Mitophagy: mechanisms, pathophysiological roles, and analysis. Biol Chem. 2012;393(7):547-564

19. Tanaka A, et al. Proteasome and p97 mediate mitophagy and degradation of mitofusins induced by Parkin. J Cell Biol. 2010;191(7):1367-1380.

20. Chen Y, Dorn GW. PINK1-phosphorylated mitofusin 2 is a Parkin receptor for culling damaged mitochondria. Science. 2013;340(6131):471-475.

21. Song M, Mihara K, Chen Y, Scorrano L, Dorn GW. Mitochondrial fission and fusion factors reciprocally orchestrate mitophagic culling in mouse hearts and cultured fibroblasts. Cell Metab. 2015;21(2):273-286.

22. Tang C, et al. PINK1-PRKN/PARK2 pathway of mitophagy is activated to protect against renal ischemia-reperfusion injury. Autophagy. 2018;14(5):880-897.

23. Rabe M, Schaefer F. Non-Transgenic Mouse Models of Kidney Disease. Nephron. 2016;133(1):53-61.

24. Kashyap S, et al. Cc12 deficiency protects against chronic renal injury in murine renovascular hypertension. Sci Rep. 2018;8(1):8598.

25. Clements M, et al. Differential Ly6C Expression after Renal Ischemia-Reperfusion Identifies Unique Macrophage Populations. J Am Soc Nephrol. 2016;27(1):159-170.

26. Pabón MA, et al. Beclin-1 regulates cigarette smoke-induced kidney injury in a murine model of chronic obstructive pulmonary disease. JCI Insight. 2018;3(18):99592.

27. Ramachandran P, et al. Differential Ly-6C expression identifies the recruited macrophage phenotype, which orchestrates the regression of murine liver fibrosis. Proc Natl Acad Sci USA. 2012;109(46):E3186-E3195.

28. Ren J, et al. Rictor/mammalian target of rapamycin complex 2 promotes macrophage activation and kidney fibrosis. $J$ Pathol. 2017;242(4):488-499.

29. Hallowell RW, et al. mTORC2 signalling regulates M2 macrophage differentiation in response to helminth infection and adaptive thermogenesis. Nat Commun. 2017;8:14208.

30. Ordureau A, et al. Defining roles of PARKIN and ubiquitin phosphorylation by PINK1 in mitochondrial quality control using a ubiquitin replacement strategy. Proc Natl Acad Sci USA. 2015;112(21):6637-6642.

31. Vives-Bauza C, et al. PINK1-dependent recruitment of Parkin to mitochondria in mitophagy. Proc Natl Acad Sci USA. 2010;107(1):378-383.

32. Wang Y, Harris DC. Macrophages in renal disease. J Am Soc Nephrol. 2011;22(1):21-27.

33. Gamboa JL, et al. Mitochondrial dysfunction and oxidative stress in patients with chronic kidney disease. Physiol Rep. 2016;4(9):e12780.

34. Chung AC, Huang XR, Zhou L, Heuchel R, Lai KN, Lan HY. Disruption of the Smad7 gene promotes renal fibrosis and inflammation in unilateral ureteral obstruction (UUO) in mice. Nephrol Dial Transplant. 2009;24(5):1443-1454.

35. Zhong X, Chung AC, Chen HY, Meng XM, Lan HY. Smad3-mediated upregulation of miR-21 promotes renal fibrosis. J Am Soc Nephrol. 2011;22(9):1668-1681.

36. Bordt EA, et al. The Putative Drp1 Inhibitor mdivi-1 Is a Reversible Mitochondrial Complex I Inhibitor that Modulates Reactive Oxygen Species. Dev Cell. 2017;40(6):583-594.e6.

37. Ishihara M, et al. Sestrin-2 and BNIP3 regulate autophagy and mitophagy in renal tubular cells in acute kidney injury. $A m J$ Physiol Renal Physiol. 2013;305(4):F495-F509.

38. Wang Y, et al. PINK1/Parkin-mediated mitophagy is activated in cisplatin nephrotoxicity to protect against kidney injury. Cell Death Dis. 2018;9(11):1113.

39. Cui J, et al. Mitochondrial autophagy involving renal injury and aging is modulated by caloric intake in aged rat kidneys. PLoS ONE. 2013;8(7):e69720.

40. Xiao L, et al. The mitochondria-targeted antioxidant MitoQ ameliorated tubular injury mediated by mitophagy in diabetic kidney disease via Nrf2/PINK1. Redox Biol. 2017;11:297-311.

41. Naik E, Dixit VM. Mitochondrial reactive oxygen species drive proinflammatory cytokine production. J Exp Med. 2011;208(3):417-420.

42. Zhou R, Yazdi AS, Menu P, Tschopp J. A role for mitochondria in NLRP3 inflammasome activation. Nature. 2011;469(7329):221-225.

43. Zhang Y, Choksi S, Chen K, Pobezinskaya Y, Linnoila I, Liu ZG. ROS play a critical role in the differentiation of alternatively activated macrophages and the occurrence of tumor-associated macrophages. Cell Res. 2013;23(7):898-914.

44. Oh MH, et al. mTORC2 Signaling Selectively Regulates the Generation and Function of Tissue-Resident Peritoneal Macrophages. Cell Rep. 2017;20(10):2439-2454.

45. Larson-Casey JL, Deshane JS, Ryan AJ, Thannickal VJ, Carter AB. Macrophage Akt1 Kinase-Mediated Mitophagy Modulates Apoptosis Resistance and Pulmonary Fibrosis. Immunity. 2016;44(3):582-596.

46. Gegg ME, Cooper JM, Chau KY, Rojo M, Schapira AH, Taanman JW. Mitofusin 1 and mitofusin 2 are ubiquitinated in a $\mathrm{PINK} 1$ /parkin-dependent manner upon induction of mitophagy. Hum Mol Genet. 2010;19(24):4861-4870.

47. Ding Y, Kim S1, Lee SY, Koo JK, Wang Z, Choi ME. Autophagy regulates TGF- $\beta$ expression and suppresses kidney fibrosis induced by unilateral ureteral obstruction. J Am Soc Nephrol. 2014;25(12):2835-2846.

48. Akchurin $\mathrm{O}$, et al. Lack of hepcidin ameliorates anemia and improves growth in an adenine-induced mouse model of chronic kidney disease. Am J Physiol Renal Physiol. 2016;311(5):F877-F889.

49. Weng SY, et al. IL-4 Receptor Alpha Signaling through Macrophages Differentially Regulates Liver Fibrosis Progression and Reversal. EBioMedicine. 2018;29:92-103.

50. Bhatia D, et al. Rituximab modulates T- and B-lymphocyte subsets and urinary CD80 excretion in patients with steroid-dependent nephrotic syndrome. Pediatr Res. 2018;84(4):520-526.

51. Iwashita H, et al. Live Cell Imaging of Mitochondrial Autophagy with a Novel Fluorescent Small Molecule. ACS Chem Biol. 2017;12(10):2546-2551.

52. Zhu L, et al. TBK-binding protein 1 regulates IL-15-induced autophagy and NKT cell survival. Nat Commun. 2018;9(1):2812. 